\title{
Cell surface nucleolin as active bait for nanomedicine in cancer therapy: a promising option.
}

Benedetta Ferrara ${ }^{a}$, Sabrina Belbekhouche ${ }^{b}$, Damien Habert ${ }^{a}$, Claire Houpe ${ }^{a}$, Benoit Vallé $e^{a}$ Sandrine Bourgoin-Voillard ${ }^{\mathrm{a}, \mathrm{c}}$, José Cohen ${ }^{\mathrm{a}}$, Ilaria Cascone ${ }^{\mathrm{a}}$, José Courty ${ }^{\mathrm{a}, \mathrm{d}}$

a Université Paris-Est, Immunorégulation et Biothérapie, INSERM U955, Hôpital Henri Mondor, 94010 Créteil, France.

${ }^{b}$ Université Paris Est Creteil, CNRS, Institut Chimie et Matériaux Paris Est, UMR 7182, 2 Rue Henri Dunant, 94320 Thiais, France

c Univ. Grenoble Alpes, Laboratory of Fundamental and Applied Bioenergetics/Prométhée proteomic platform, UGA-Inserm U1055-CHUGA, Grenoble

${ }^{d}$ Corresponding author : José Courty at: Université Paris-Est, Immunorégulation et Biothérapie, INSERM U955, Hôpital Henri Mondor, 94010 Créteil, France, courty@u-pec.fr 


\begin{abstract}
Conventional chemotherapy used against cancer is mostly limited due to their non-targeted nature, affecting normal tissue and causing undesirable toxic effects to the affected tissue. With the aim of improving these treatments both therapeutically and in terms of their safety, numerous studies are currently being carried out using nanoparticles as a vector combining tumour targeting and carrying therapeutic tools. In this context, it appears that nucleolin, a molecule over-expressed on the surface of tumour cells, is an interesting therapeutic target. Several ligands of various origins, such as AS1411, the F3 peptide and the multivalent pseudopeptide N6L have been developed and studied as therapeutic tools against cancer. Over the last ten years or so, numerous studies have been published demonstrating that these antagonists can be used as tumour targeting agents with nanoparticles from various origins. Focusing on nucleolin ligands, the aim of this article is to review the literature recently published or under experimentation in our research team to evaluate the efficacy and future development of these tools as anti-tumour agents.
\end{abstract}

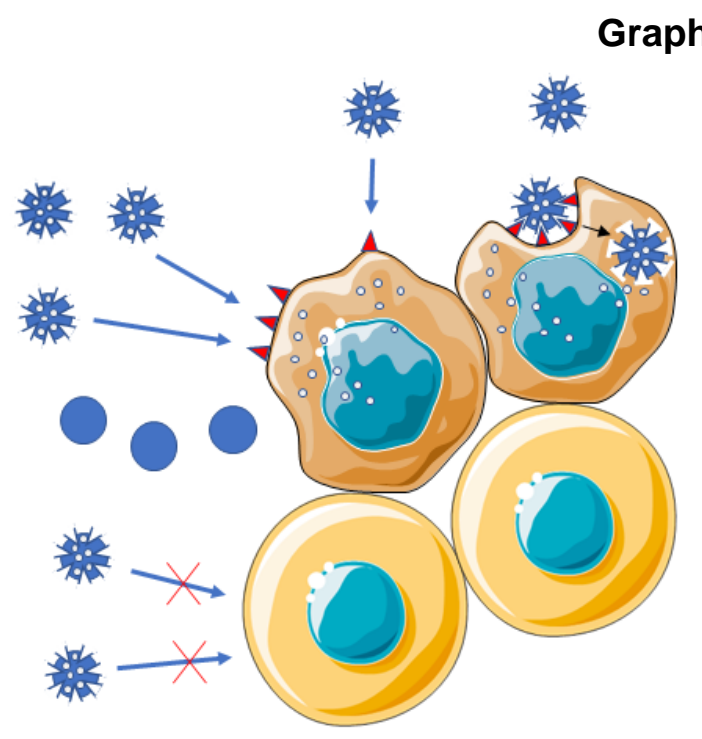

Non-tumour cell

Tumour cell

资 Ligands: nucleolin antagonist

Tumour cell target : cell surface nucleolin

$\because$ Cytotoxic molecules

Nanoparticles

Functionalized nanoparticles 


\section{Introduction}

A growing body of evidence has reported that nanomedicine could be one of the tools that can revolutionize the future of medicine with great potential in drug delivery ${ }^{1}$. This observation is especially true in cancer treatment where used drugs are often toxic for non tumor cells with many side effects ${ }^{2-9}$. One of the main concept of the use of nanotechnology-based therapies is to enhance the accumulation of drugs in tumours without affecting the normal tissues. The ultimate challenge is to design nanometric structures that display anti-tumour effect and/or loaded with cytotoxic molecules targeting tumour cells and to deliver the therapeutic molecules without reaching normal cells. In addition, this nanometric structure must be not cytotoxic, able to delivery cytotoxic molecules specifically to the tumour cells and biodegradable or not but eliminated from the body.

Several studies have reported that the used of nanoparticles functionalized with tumour target molecules could surmont this challenge. The targets tested generically referred to as "tumourassociated antigens" 10, are mostly derived from over-expressed or genetically altered molecules and are essential for the survival and proliferation of tumour cells. Several types of molecules can be considered, those derived directly from tumour cells such as HER2 ${ }^{11,12}$, FLT3 ${ }^{13}$, EGFR ${ }^{14}$, mTOR $^{15}$ or from the tumour stroma such as VEGF/VEGFR ${ }^{12}$, mTOR $^{16}$ or those related to immunocheck points ${ }^{9,17,18}$. For several years our research team has focused on the use of nucleolin as an agent that can be used as a tumour cell targeting molecules. Indeed, it is now well demonstrated that contrary to normal cells, tumour cells overexpress this molecule on the surface of their plasma membrane, thus allowing specific targeting of these cells. Thus, within the framework of these studies, we have developed a nucleolin ligand of peptide origin which can be used to target tumour tissue. This pseudopeptide, called N6L, does not present toxicity at the therapeutic dose and has been the subject of a phase $\mathrm{I} /$ /la clinical study.

This review gives an update on studies related to nanomedicine in cancer therapy focusing on the use of nucleolin as a therapeutic target.

\section{Nanomedicine as therapeutic and diagnostic strategy for cancer}

Cancer is the second leading cause of mortality worldwide, with 9.6 million deaths in 2018. Globally, the prevalence of cancer has constantly increased. About $70 \%$ of deaths due to cancer occur in low- and middle-income countries ${ }^{2}$. Men display the prevalence of cancer in the prostate, lung, colon and rectum and also bladder, while women are mostly affected by cancer of breast, lung, colon and rectum, uterus and thyroid ${ }^{19}$. In almost all cases, cancer derives from an accumulation of successive mutations in genes implicated in the control of cell proliferation, survival and death ${ }^{20}$. Several carcinogenic chemical compounds and physical factors, such as viruses, bacteria and radiation, lead to these mutations in proto-oncogenes 
and tumour suppressor genes ${ }^{21}$. Most cancers are also developed because of behavioural and dietary risks, such as obesity, lack of physical activity, use of tobacco and alcohol ${ }^{22}$. During their progression, tumours become very heterogeneous and are characterized by a variety of molecular and physical features conferring different response to therapeutic treatments ${ }^{23}$.

Therefore, it is important to develop novel therapeutic strategies which could specifically target each cancer type. After surgery, which is the first and most effective treatment for localized primary tumours, chemotherapy is conventionally used for the systemic diseases ${ }^{3}$. However, the low specificity of chemotherapeutic drugs requires administering at high doses, leading to severe side effects limiting their use in many patients. In the last years, a growing body of scientific evidence gave life to the era of "targeted therapy" ${ }^{19}$. Indeed, nowadays one of the main objectives of cancer research is to identify the best way to target only the desired tumour site.

In recent years, nanomedicine has acquired many interests since it gives a solution for the lack of specific systems for cancer therapy ${ }^{24,25}$. Nanoparticles (NPs) can specifically accumulate in the tumour tissue due to the enhanced permeability and retention (EPR) effect ${ }^{26,27}$. Indeed, the leaky vasculature and impaired lymphatic drainage of tumour tissues allow these nanosized systems $(1-100 \mathrm{~nm} \text { in size })^{28}$ to reach the neoplastic site ${ }^{29}$. NPs are able to incorporate hydrophilic and lipophilic drugs providing a prolonged and controlled drug release into the target cell. The biocompatible NPs allow the administration of conventional anticancer drugs by overcoming some of their limits, such as low specificity, bioavailability and solubility ${ }^{30}$. Among the different types of NPs, inorganic NPs are mostly employed as contrast agents for the diagnosis ${ }^{30}$. Indeed, they can provide an encouraging solution as precursors for targeted contrast agents in the methods of magnetic resonance imaging (MRI) and computed tomography (CT). Inorganic NPs include quantum dots, superparamagnetic iron oxide NPs (SPIONs) and gold NPs which are very important for electrical properties and low toxicity. Organic NPs, include liposomes, micelles, cyclodextrin-NPs, solid lipid NPs, and are mostly used for the delivery of drugs. Polymeric NPs are constituted of biocompatible or natural polymers, such as poly (lactide-co-glycolide), poly ( $\varepsilon$-caprolactone), chitosan, alginate and albumin ${ }^{31}$.

Since 1995, many nanoformulations have been approved by FDA and are commonly used in the clinical practice. They reported a reduced toxicity and an increased efficacy when compared to conventional formulations ${ }^{32}$.

NPs can be functionalized with ligands that recognize cell surface components or subcellular sites. These ligands are constituted by small molecules, polypeptides, protein domains, antibodies and nucleic acid-based aptamers ${ }^{32}$.

Accumulating evidence validates cell surface nucleolin (NCL) as a potential target for the treatment of cancer which could bind to several ligands ${ }^{33}$. In this context, NPs functionalized 
with molecules against NCL have been developed and revealed promising results in terms of efficacy and safety. This review has the aim to point out the results achieved with different types of NPs targeting cancer cells by means of anti-NCL ligands and DNA or other systems

\section{Nucleolin as a tumour cell target}

NCL is a $77 \mathrm{kDa}$ phosphoprotein ${ }^{34}$ which represents one of the major constituents of the nucleolus in mammalians, accounting for $10 \%{ }^{35,36}$ in the protein component. It is reported to be present also in the nucleus, in the cytoplasm and on the plasma membrane. NCL is constituted of 3 main structural domains ${ }^{37}$ : the $\mathrm{N}$-terminal domain which is rich in acidic glutamate/aspartate sequence repeats and represents the site of protein-protein interactions 37, 38; the central domain which contains 4 RNA-binding domains that enable the interactions with many nucleic acids ${ }^{39}$; the C-terminal region which is rich in glycine, arginine and phenylalanine residues and is susceptible to post-translational methylation modifications ${ }^{40}$. By interacting with other proteins and nucleic acids, NCL takes part to several critical processes such as the maturation of pre-rRNA, the transcription of rDNA and the assembly of the ribosomal subunits during ribosome biosynthesis. By associating with the actin cytoskeleton and various membrane-anchored proteins, NCL is also involved in the process of endocytosis and in the shuttling of proteins back and forth between nucleus and cytoplasm ${ }^{41}$. Moreover, cell surface NCL plays an important role in several pathways related to the cell cycle, cell proliferation and apoptosis ${ }^{42,43}$.

The biological functions of NCL are important for cell homoeostasis but also contribute to the development of pathological conditions. In recent years, the association of NCL with cancer has been largely investigated and this protein reported to be involved in tumorigenesis 44,45 . Indeed, the expression of NCL is significantly enhanced in several solid tumours such as pancreatic cancer, thyroid cancer, breast cancer, colorectal cancer, prostate cancer, gastric cancer, melanoma, glioma, hepatocellular carcinoma, but also in leukaemia ${ }^{46-57}$. The overexpression of NCL and its accumulation of NCL at the cell surface are related with a poor prognosis in the majority of cancers ${ }^{58,59}$. An aberrant up-regulation of NCL is responsible for the increased rRNA synthesis and ribosome assembly which must be implemented in highly dividing cells ${ }^{60}$. Moreover, NCL can interact with different mRNAs which can be implicated in the regulation of apoptosis and cell survival ${ }^{61}$. Indeed, it can increase the levels of BCL-2, which acts as an inhibitor of apoptosis, in the tumour cells by binding to the BCL-2 AU-rich elements (AREs) ${ }^{62}$. This region is responsible for the stability of the BCL-2 mRNA; therefore $\mathrm{NCL}$ can enhance the resistance to apoptosis in cancer cells ${ }^{62}$. By binding to the 5 ' UTR of TP53 mRNA, NCL can also induce the translation of AKT1 and cyclin I, which promote cell survival ${ }^{62}$. 
$\mathrm{NCL}$ is also overexpressed in angiogenic and endothelial cells and can regulate the process of angiogenesis which is crucial for cancer progression by enhancing the expression of vascular endothelial growth factor (VEGF) ${ }^{63,64 .}$

On cell surface NCL can interact with several ligands such as CXCL12/CXCR4, activating its signalling which influences growth, migration and invasiveness of cancer cells, and hepatocyte growth factor (HGF) involved in the mechanisms of metastasis and cell adhesion ${ }^{65-67}$.

Another factor is EGFR, which is implicated in tumour proliferation, regulation of apoptosis, angiogenesis and metastasis. NCL can link to EGFR mRNA inducing its pathway thus increasing cell viability, colony forming ability and migration ${ }^{68}$. Moreover, NCL exerts a synergic effect with activated $\mathrm{H}$-Ras and EGFR in promoting the cell transformation ${ }^{69}$.

By interacting with TGF-beta receptor I, on the cell surface, NCL can promote and regulate the pathway of TGF- $\beta$ which is one of the most important factors involved in inflammatory response, angiogenesis and remodelling of the extracellular matrix (ECM) ${ }^{58,68}$.

$\mathrm{NCL}$ is abundantly present in haematopoietic stem/progenitor cells (HSPCs) where it promotes the responsiveness to Wnt-3a ${ }^{70}$. It also induces the signalling of Wnt/GSK3 and BMP, which contribute to the regulation of HSPCs and is associated with leukaemic stem cells in some leukaemias ${ }^{71-73}$.

Since NCL is highly expressed in the cell surface of various cancer cells and in angiogenic endothelial cells, it could represent an important biomarker for the diagnosis or a target for potential therapy directed against both these cell types $46,61,74,75$. Indeed, its position on the cell surface can be exploited for the binding of anticancer drugs which enter the tumour cell affecting its function ${ }^{76,77}$. NPs loaded with anticancer drugs can constitute a ligand for NCL which allows their transfer through the cell and to the nucleus.

Besides, since NCL is up-regulated in different cancer types, therapeutic strategies will not be limited to a specific group of patients. Various types of formulations have been proposed for the delivery of drugs targeting NCL ${ }^{59}$. This work presents a variety of therapeutic tools developed in recent years by investigating new molecules for the target therapy of NCL and implementing new strategies of drug delivery.

NCL holds the attention as a target for cancer therapies accounting to its implication in tumorigenesis but also and mainly for its increase expression in cancer cells ${ }^{59}$. This research topic has been getting more and more attention in recent years. As shown in figure 1, publications and citations based on keywords "nucleolin, nanoparticles and cancer" have increased for the past of the decade.

\section{AS1411 aptamer as a NCL-ligand}

The aptamers can bind a wide range of molecular targets due to their three-dimensional folding. AS1411 aptamer is capable of binding NCL, and preferentially its external domain on 
the cell surface, with high affinity and specificity ${ }^{78,79}$. AS1411 is a 26-base DNA aptamer with a G-quadruplex structure which gives stability against the serum nucleases and the $\mathrm{pH}$ fluctuations leading to increase of the cellular uptake efficacy ${ }^{80,81}$. This aptamer is heat stable, non-immunogenic and resistant to DNase/RNase degradation in serum-containing medium. AS1411 aptamer is the most advanced and the first tested aptamer in cancer therapy ${ }^{81,82}$ and it can potentially be used in clinics since it presents a good safety profile and the ability to produce responses in patients with intractable tumours, as reported by phases 1 and 2 clinical trials. Therefore, different drug delivery systems have been investigated to develop more specific therapies through the binding of AS1411 aptamer to NCL ${ }^{83,84}$. There is scientific evidence reporting the ability of AS1411-conjugated NPs to circumvent drug-resistance pumps, to pass the blood-brain barrier and to reduce the toxicity towards normal tissues ${ }^{85-87}$. Moreover, AS1411 revealed a potential use as a targeting agent for imaging applications also being incorporated into particles designed for theranostic use or multimodal imaging ${ }^{63,88}$.

\subsection{AS1411 aptamer-conjugated liposomes}

Among the different nanocarriers developed to specifically target NCL through the binding of AS1411, liposomes have been largely investigated since they constitute an ideal vehicle for many molecules and drugs. Liposomes are vesicles of spherical shape composed of a lipid bilayer surrounding an internal aqueous cavity ${ }^{89}$. The structural components of liposomes include phospholipids or synthetic amphiphiles incorporated with sterols for modifying the membrane permeability. Different types of liposomes can be developed by altering their size, composition and charge ${ }^{90}$. Drugs which display a different solubility can be delivered by liposomes thus providing a stable and more efficient therapeutic system ${ }^{91}$. Several liposomal formulations have been accepted for the clinical use, such as Doxil ${ }^{\circledR}$, approved in 1995 by FDA for the delivery of doxorubicin in ovarian cancer, or DaunoXome ${ }^{\circledR}$, approved in 1996 for carrying daunorubicin in HIV-associated Kaposi's sarcoma (Barenholz 2012). Since they improve the pharmacodynamics and pharmacokinetics of conventional drugs, many other liposomal formulations are now in different phases of clinical investigation ${ }^{92}$. Liposomes can be associated with ligands to improve their specificity.

Among all the liposomal systems linked to AS1411 and used for carrying anticancer drugs directly to the tumour site, several nano-formulations presenting different peculiarities are herein pointed out in the present work.

PEGylated cationic liposomes conjugated to AS1411 were proposed for delivering anti-BRAF siRNA in melanoma ${ }^{51}$. Indeed, the silencing of BRAF gene, which is implicated in the melanocytic neoplasia formation, may provide a promising therapy for this cancer. Liposomes were chosen for carrying the siRNA since they constitute a successful system for drug delivery 
and many of them were approved by FDA for their application in the clinical practice ${ }^{92}$. Moreover, the aptamer AS1411 was conjugated to the liposomal formulation to reach a specific effect against NCL expressed on the surface of A375 melanoma cells. The results obtained in vitro indicate a strong efficacy of this nano-formulation on A375 cell line, where a significant silencing of BRAF gene was demonstrated with real-time PCR and a high inhibitory activity was assessed by cell viability assay. The in vivo study confirmed these findings reporting a high and constant distribution of liposomes at the tumour site and a reduction in the expression of BRAF mRNA, after iv injections in a xenograft model of $A 375{ }^{51}$.

AS1411-linked liposomes were also proposed for the delivery of miRNA-29b in ovarian cancer. The miR-29b binds to DNA methyltransferases controlling the demethylation of DNA and thus leading to the inhibition of cancer cell growth. The cationic liposomes allow loading the miRNA on the surface due to physical forces ${ }^{93}$. In order to achieve a selective target to the tumour site, miR-29b loaded liposomes (LP-miR) were conjugated with aptamer AS1411. Flow cytometry and confocal laser scanning microscopy (CLSM) evidenced a successful targeting of the A2780 ovarian cancer cells through the binding of the aptamer to NCL. An antiproliferative effect of the LP-miR formulation was assessed in vitro by MTT assay on A2780 cell line revealing a concentration-dependent cytotoxicity. To confirm this result, the live/dead assay showed a significant decreased in green fluorescence which is representative of decreased cell viability in cells treated with LP-miR, while the apoptotic assays indicated a high presence of apoptotic cells. Therefore, this novel formulation may potentially constitute a new pharmaceutical tool for the therapy of ovarian cancer ${ }^{94}$.

This system of delivery is highly successful even when used to incorporate chemotherapeutic drugs ${ }^{95}$. Doxorubicin (DOX) is known to be an effective anticancer drug, but its efficacy is limited by the resistance displayed by cancer cells due to efflux pumps and by its high cardiotoxicity ${ }^{96}$. A nano-system to overcome these problems was developed by using thermoresponsive liposomes which can control and rapidly trigger the drug release. In order to efficiently encapsulating DOX, ammonium bicarbonate $(A B C)$ was incorporated in the liposomal system 97. Local hyperthermia, induced by ultrasound energy, microwave, radiofrequency or magnetically, allows $A B C$ to convert the liposome membranes into permeable defects which can easily accumulate at the tumour interstitium. In addition, liposomes containing DOX hydrochloride and $A B C$ were functionalized with the aptamer AS1411 for targeting MCF-7 breast cancer cells. The formulation was characterized using dynamic light scattering (DLS) and ultrasonography. The in vitro release evaluation showed that AS1411-functionalized liposomes were stable at body temperature, while they released immediately DOX when the temperature increased. DOX-loaded AS1411-liposomes 
treated cells revealed a high fluorescent intensity by fluorescence microscopy, reporting a substantial cellular uptake, confirmed by CLSM and flow cytometry which revealed a significant accumulation of DOX in the cell nuclei. Finally, in vivo results obtained on tumour bearing nude mice demonstrated that DOX-loaded AS1411-liposomes with mild local heating could inhibit the tumour growth reducing cardiotoxicity and other side effects. This finding represents a promising therapeutic strategy for the treatment of breast cancer ${ }^{98}$.

In the context of breast cancer, Yu S et al. developed a system based on the combination of paclitaxel (PTX) and a siRNA directed against polo-like kinase 1 (PLK1), a highly conserved serine/threonine protein kinase overexpressed in breast cancer causing an abnormal cell proliferation. The idea was to simultaneously administer one of the main chemotherapeutic drugs used in breast cancer but which its use is limited by very poor water solubility, and a silencer of a single target expressed on breast cancer cells to provide a complete and selective treatment. Moreover, liposomes were chosen to carry PTX and the siRNA for improving their stability and they were conjugated with AS1411 aptamer. After incorporating the lipid DOPE for enhancing the membrane fusion and transfection effectiveness and adding the sphingomyelin containing an amide-related fatty chain for reducing biodegradation, the stability of the nano-formulation was verified by TEM and serum stability assay. MCF-7 cells overexpress NCL and were then chosen as model for detecting the specificity of AS1411/LipoPTX-siPLK1. It is reported to specifically bind to the MCF-7 cell surface transporting both PTX and SiPLK1 inside the cell. Frozen tumour tissue sections from MCF-7 xenograft mice treated with AS1411/Lipo-PTX-siPLK1 indicated a high accumulation of liposomes at the tumour site. The tumour growth of treated mice was reduced and their survival was increased. Besides, an increase of apoptosis and an inhibition of angiogenesis were revealed with TUNEL assay and CD31 staining, respectively. Altogether, the results of this study assert that the combination of PTX and PLK1 siRNA delivered by AS1411-functionalized liposomes exert a synergistic effect in breast cancer ${ }^{99}$.

\subsection{AS1411 aptamer-conjugated PLGA-NPs}

In the challenge of developing nano-vehicles for targeted drug delivery to cancer cells overexpressing NCL, various types of poly (lactic-co-glycolic acid) (PLGA)-based NPs linked to AS1411 have been investigated.

PLGA is a biocompatible polymer which has been largely investigated for its application as a drug delivery system. It allows a sustained and prolonged drug release and a long-term stability of loaded molecules ${ }^{100-102}$. Furthermore, PEG is a FDA-approved polymer used for the surface coating of a large number of NPs, in a process called PEGylation which protects NPs from the immune system. The PEGylated NPs show higher biocompatibility and improved cytoplasmic transport than the non-PEGylated NPs ${ }^{103-105 .}$ 
In the work of Alibolandi M et al. ${ }^{106}$, a formulation based on polyethylene glycol (PEG)-PLGA functionalized with AS144 aptamer was proposed for transporting gemcitabine (GEM) to NCLoverexpressing non-small cell lung cancer (NSCLC) cells. GEM is a powerful anticancer drug used for the treatment of NSCLC, which constitutes one of the main and aggressive types of lung cancer ${ }^{107}$. The delivery of GEM by nano-vehicles improves its half-life and efficacy. TEM images and DLS measurements allowed characterizing GEM-loaded PEG-PLGA nanopolymers. Gel electrophoresis and UV spectroscopy revealed the efficacy of conjugation with DNA AS1411 aptamer. The aptamer functionalization on the NP surface enables the cellular uptake which was proven to selectively occur in NCL-overexpressing A549 cells, as demonstrated by fluorescent microscopy. This last technique showed that Apt-GEM-NPs mostly distributed inside the cells at $37^{\circ} \mathrm{C}$. Flow cytometry analysis reported that AS 1411 aptamer can increase the uptake of Apt-NPs inside A549 cells, but not in the pretreated cells with the free aptamer. Consistently, in vitro obtained results on cell viability revealed an $\mathrm{IC}_{50}$ value for Apt-GEM-NPs much lower than GEM-NPs, while these values for Apt-GEM-NPs and GEM-NPs in free aptamer-treated A549 was similar. In conclusion, the incorporation of GEM in AS1411 aptamer-functionalized NPs selectively increase the effectiveness of GEM in A549 NSCLC.

PEGylated PLGA-NPs decorated with AS1411 aptamer were also proposed for delivering cisplatin (CIS) to ovarian cancer (OC) cells ${ }^{108}$. Indeed, CIS is one of the first-line therapies against $\mathrm{OC}$ but it needs to be administered in combination with other anti-cancer molecules for prolonging the patients' survival due to the development of chemoresistance in OC cells ${ }^{109}$. Since the role played by miR-21 in chemoresistance and tumour progression has been largely investigated ${ }^{110}$, the permanent inhibition of this miRNA with an anti-miR-21 agent in combination with the conventional chemotherapy may be a successful strategy for improving OC treatment. In the work presented by Vandghanooni et al ${ }^{108}$, AS1411 aptamer-conjugated PEG-PLGA containing anti-miR-21 (Ap-anti-miR-21-NPs) and containing CIS (Ap-CIS-NPs) were designed. Since antisense miRs easily undergo enzymatic degradation, Ap-anti-miR-21NPs were formulated for protecting from nuclease degradation ${ }^{111}$. The prepared nanosuspensions were characterized by nano-ranged size, spherical shape and narrow distribution profile. They displayed a fast release within the cells in the first $8 \mathrm{~h}$ becoming sustained for 72h. The binding of AS1411 Ap to A2780 and A2780-res cells which overexpress NCL resulted higher than the one of $\mathrm{CHO}$ cells (which do not express $\mathrm{NCL}$ ) thereby allowing assessing that the NCL-mediated endocytosis is involved in the cellular uptake of AS1411 Ap-NPs. MTT assay revealed that miR-21-inhibited A2780-res cells displayed a significant inhibition of cell viability upon treatment with Ap-CIS-NPs, demonstrating the synergistic effect of the two drugs. 
The results obtained in the reported study support previous works showing the strong antiapoptotic effect of anti-miR-21 loaded PLGA-NPs in combination with chemotherapeutic drugs 100. Moreover, DAPI staining and DNA fragmentation assay assessed significant nuclear morphological changes induced by apoptosis in A2780 cells treated with Ap-CIS-NPs and A2780-res cells treated with Ap-anti-miR-21-NPs. Mir-21-inhibited A2780-res cells displayed an enhanced cell growth arrest and a high frequency of apoptotic cells compared to the uninfected cells upon treatment with Ap-CIS-NPs. In conclusion, the pretreatment of OC cells with Ap-anti-miR-21-NPs together with nano-systems delivering chemotherapeutic drugs may be promising for the treatment of this cancer.

In the work of Mosafer et al ${ }^{112}$, PLGA-based NPs targeted with AS1411 aptamer and loaded with superparamagnetic iron oxide nanoparticles (SPIONs) and DOX were successfully developed against murine C26 colon carcinoma cells in order to propose a novel theranostic system. A co-precipitation method was used to synthesize the SPIONs as diagnostic agents and their incorporation in the nano-system, as well as the one of DOX, was based on a multiple emulsion solvent evaporation method ${ }^{113}$. The physico-chemical characteristics of the prepared nano-formulations, such as particle size distribution, saturation magnetization and drug loading capacity were investigated and reported an efficient co-incorporation of DOX and SPIONs and a constant drug release profile. A narrow particle size distribution and mono-modal population of the NPs were pointed out by DLS results, and the morphology of NPs by AFM revealed a spherical shape and smooth surface (NPs size around $130 \mathrm{~nm}$ ). The best in vitro release of DOX was observed in PBS at $\mathrm{pH} 7.4$, where an initial low burst release was followed by a slower DOX release for 20 days. Flow cytometry images revealed that the cellular uptake of Apt-NPs is dependent on the interaction Apt-NCL on C26 cells surface and the binding of Apt to NPs increased the cellular uptake of DOX. Apt-NPs loading DOX displayed a higher antiproliferative effect in vitro on C26 cells in a dose-dependent manner. Moreover, in vivo experiments were carried out on xenograft colon carcinoma mice subcutaneously injected with C26 cells. The survival rate was higher in the animals treated with DOX-loaded Apt-NPs with respect to mice receiving free DOX, while the tumour size was significantly reduced in the first group of mice. These results may be important for developing tumour-targeted NPs for therapeutic applications. Finally, magnetic resonance imaging (MRI) of C26 colon carcinoma after 1-6 h post-injection showed a strong accumulation and penetration of Apt-NPs in the tumour site revealing the importance of the Apt-NPs as potential theranostic agents.

\subsection{Other types of AS1411 aptamer-conjugated NPs}

In the work of Sun XY et al ${ }^{114}$, a hybridized DNA structure was designed and conjugated with superparamagnetic iron oxide NPs (SPION) to generate a multifunctional nanoformulation 
carrying a chemotherapeutic drug and a photosensitizer molecule ${ }^{115,116}$. SPION has important magnetic property but it presents a lack of active targeting effect which limits its application. Nucleic acids function as attractive tools being largely employed in precise medicine in recent years ${ }^{117,118}$. In this study, AS1411 aptamer was chosen and linked to the surface of SPION for the targeting surface NCL and the photosensitizer 5-10-15-20-tetrakis (1-methylpyridinium4-yl) pophyrin (TMPyP) was loaded to the system ${ }^{119,120}$. Moreover, also a cytosine-rich hairpin DNA loading daunorubicin (DNM) was added at the SPION surface for controlling the drug release in the tumour microenvironment. The characterization of the nanoformulation revealed the stability of the designed drug delivery system called TMPyP DNM Apt-gc34@SPION NPs and its biosafety for circulating in the blood. The $\mathrm{pH}$-sensitive release property of TMPyP DNM Apt-gc34@SPION NPs reported minimizing premature release of DNM and facilitates the active drug release at the acid target tissues. The drug binding and release demonstrated to be continuously cycled when the $\mathrm{pH}$ varies between 7.4 and 5.6. The molecular docking showed the binding of the drug molecules to Apt-gc34 DNA and indicated a stable drug delivery by Apt-gc34. However, the interactive conformations of the drugs and DNA structure reported being intense ${ }^{114}$. Regarding in vitro tests, the wound healing assay was carried out on A549 lung cancer cell line to assess that TMPyP DNM Apt-gc34@SPION NPs can influence and reduce cell migration and invasion. In vitro magnetic targeted experiment was performed to evaluate the magnetic targeted property of SPION and, after cell incubation with TMPyP DNM Apt-gc34@SPION NPs, most of the cells in the magnetic region were died, underlying the fact that magnetic NPs can successfully distribute in the targeted area under magnetic guidance. CLSM reported the intracellular release of the nano-formulation showing that DNM was released in acid microenvironment of cancer cells. Further, NCL-blocked cells displayed much lower ROS production after treatment with TMPyP DNM Apt-gc34@SPION NPs with respect to blocked cells. Results of MTT assay demonstrated that cell viability was highly reduced in NCL-non-blocked cells indicating that AS1411 structure plays an important role for the cellular internalization of the designed drug delivery system resulting in a higher cell inhibition ${ }^{82,121}$. A significant reduction in cell growth under the visible light was observed and was due to the synergistic effect of DNM and TMPyP. Further, the level of apoptosis-related protein bcl-2 and c-myc rapidly decreased after the treatment with TMPyP DNM Aptgc34@SPION NPs. In conclusion, the results presented herein suggest the important role of this designed new delivery system as a successful tool for a targeted anticancer drug-deliverybased therapy.

Taghavi $S$ et al ${ }^{122}$ proposed a delivery system based on AS1411-functionalized carbon nanotubes (CNTs) for the treatment of gastric cancer cells. In their study, single-walled CNTs were conjugated to the branched polyethylenimine (PEI) and polyethylene glycol (PEG) and the resulting formulation, SWCNT-PEG-10-10\%PEI, was loaded with a shRNA for Bcl-xL 
which exerts a strong anti-apoptotic effect being crucial for tumour development ${ }^{123}$. CNTs present useful physicochemical properties, a nano-needle structure and can easily enter cells through endocytosis ${ }^{124,125}$. Some limitations of CNTs include a negligible solubility and toxicity, but chemical modifications can improve their pharmacokinetics ${ }^{126}$. SWCNT-PEG-10-10\%PEI NPs modified with AS1411 were used herein for delivering Bcl-xL shRNA and DOX to AGS cancer cells and L929 normal cells. The covalent conjugation of AS1411 aptamer to NPs was confirmed by agarose gel electrophoresis and the transfection assays revealed that AptSWCNT-PEG-PEI was highly specific for the tumour cells, validating the significant role of AS144 aptamer in the enhanced gene delivery to cancer cells. Western blot analysis reported that the levels of $\mathrm{Bcl}-\mathrm{xL}$ protein decreased after $72 \mathrm{~h}$ post transfection with $\mathrm{Bcl}-\mathrm{xL}$ shRNA. The expression levels of $\mathrm{pBcl}-\mathrm{xL}$ were investigated and the obtained results revealed that $\mathrm{Bcl}-\mathrm{xL}$ shRNA is able to down-regulate the expression of $\mathrm{Bcl}-\mathrm{xL}$ protein in AGS and L929 cells treated with SWCNT-PEG-PEl/pBcl-xL shRNA complex, while Bcl-xL expression did not change in L929 treated with SWCNT-PEG-PEI-Apt/pBcl-xL shRNA assessing that the addition of AS1411 aptamer to NPs could not enhance the Bcl-xL expression, and thus the transfection efficiency which is due to the absence of NCL on the surface of L929 cells. The potential cytotoxic synergy of DOX and Bcl-xL shRNA towards AGS cells was evaluated by MTT assay and showed that the combination of pBcl-xL shRNA/SWCNT-PEG-10-10\%PEI-Apt/DOX decreased cell viability (which was $77 \%$ with only DOX) to $45 \%$. Moreover, this combination indicated significant additional cytotoxicity compared to treatments using only shRNA, DOX/SWCNT-PEG-10-10\%PEI-Apt and pshRNA scrambled/SWCNT-PEG-10-10\%PEIApt/DOX. In conclusion, a targeted co-delivery system for gastric cancer cells was developed and displayed a precise targeting effect with great potential of entering cells through both aptamer-mediated binding to NCL and nano-needle structure of SWCNT.

Since NPs functionalized with bioactive ligands constitute a promising tool to mediate the transportation of drugs across the blood-brain barrier (BBB) by receptor-mediated transcytosis, a novel nano-formulation based on lipid NPs functionalized with the angiopep-2 (ANG-2) and AS1411 aptamer was investigated by Wang $S$ et al 2018 for delivering DOX in glioma ${ }^{127}$. The cascade dual-targeting strategy has been demonstrated to be a viable approach for glioma therapy, where the first ligand overcomes the BBB barrier and the second one specifically targets brain cancer cells ${ }^{128,129}$. ANG-2 is a peptide which binds LRP expressed on the membrane of BBB and it has been largely investigated for allowing the transportation of NPs through the BBB ${ }^{130,131}$. The aptamer AS1411 was utilized for achieving an accurate glioma cells targeting. The dual targeting delivery system was constructed using lipid capped polymer NPs with PLGA as the core, which can enhance hydrophobic drugs encapsulation ${ }^{131}$, and lipids (lecithin and DSPE-PEG ${ }_{2000}-\mathrm{COOH}$ ) as the shell. The PEG chain protects the internal PLGA from hydrolysis and the carboxyl group PEG constitutes an anchor for conjugating Apt 
and ANG-2. Concerning the preparation method, PLGA and DOX were dissolved in the organic phase of methanol/chloroform and then added dropwise to the aqueous phase of lipid solution ${ }^{130}$. The ligands were conjugated to the free lipid first prior to the NPs preparation. TEM indicated the NPs spherical shape with homogeneous size. After encapsulation into NPs, a remarkable sustained DOX release was observed; an initial drug release from NPs occurred in the first 24 hours, followed by a slower rate. Cell uptake experiments showed that after incubating with unmodified NPs, only moderate DOX red fluorescence was observed inside BCEC glioma cells and a similar uptake was seen for Apt modified NPs. After incubating with ANG-2, a stronger red signal was observed inside cells suggesting a role of ANG-2 in allowing cell uptake. Flow cytometry results revealed that ANG-2 decorated NPs and also Apt and ANG2 dual decorated NPs displayed fluorescence significantly stronger than those treated with unmodified NPs. Compared to ANG-2, AS1411 reported a better-binding affinity to C6 NCLoverexpressing glioma cells ${ }^{132}$. The dual modification of NPs enhanced cell fluorescence, suggesting a synergistic effect of the two ligands. For studying the BBB penetration ability in vitro, transepithelial electrical resistance (TEER) was measured as a function of time after BCECs seeding and adding of the different NPs to the transwell chamber. For all groups the DOX amount was time-dependent, with a higher accumulation of DOX loaded in NPs. Moreover, with ANG-2 decoration of NPs, the transportation was remarkably higher. Finally, the in vitro cytotoxicity on C6 cells reported to progressively increase with higher DOX concentration after $48 \mathrm{~h}$ incubation and after Apt modification the cytostatic activity of NPs was strongly enhanced.

In the work of $\mathrm{Li} \mathrm{Y}$ et al., ${ }^{133}$, the authors presented a simple method to produce aptamertargeting organic fluorescence NPs. Indeed, fluorescence imaging systems are important for early cancer detection, assessing tumour margins and monitoring response to therapy ${ }^{120,134}$. However, many fluorescent NPs are composed of inorganic species and even some toxic heavy metal cations and it limited their clinical use ${ }^{135,136}$. Aptamer-functionalized organic NPs were proposed and rubrene, a highly emissive organic semiconductor, was chosen for their fabrication. The hydrophobic lipid segments were embedded in hydrophobic rubrene aggregates, while the hydrophilic PEG chains extend into the aqueous phase to provide water dispensability and colloidal stability. Incorporating AS1411 aptamer allowed achieving targeting functionality for facilitating the translation of organic light-emitting materials to clinical practice. Cholesterol was covalently linked to aptamer as an anchor to be inserted into the lipid membrane. The Apt-Rub NPs were prepared by mixing the hydrophobic rubrene and lipid with the cholesterol-tagged aptamer. The maximum emission of rubrene NPs appeared at $560 \mathrm{~nm}$, with an intense emission tail extended to $650 \mathrm{~nm}$, which held advantages of low optical absorption and autofluorescence from biomolecules. NPs were fabricated using 75 ug as the optimal rubrene amount and the TEM images revealed that the as-prepared Apt-Rub NPs 
exhibited a spherical shape with an average size of $-40 \mathrm{~nm}$ and a surface negative charge. The fluorescence and hydrodynamic diameter stability of NPs were evaluated and showed that all NPs were stable without aggregation. MCF-7 (human breast cancer cell line), C6 (rat glial tumour cell line) and A549 (human lung cancer cell line) were highly fluorescent after treatment with Apt-Rub NPs, as reported by fluorescence microscopy and flow cytometry, while 293T normal cells (human embryonic kidney cell line) did not display fluorescence intensity. The flow cytometric analysis revealed that the Apt-Rub NPs were specifically internalized by cells overexpressing NCL. The results on cell viability showed that Apt-Rub NPs displayed no cytotoxicity toward all the cell lines. For evaluating long-term toxicity of NPs, the weight fluctuation and behaviours of the mouse after administration did not display abnormalities. Altogether, these NPs resulted suitable for long-term tracking and imaging, being promising in biomedical applications.

\section{F3 peptide as a NCL-ligand}

F3 is a 30-34-amino acid fragment of a high mobility group protein, HMG2N and was discovered by using phage-displayed cDNA libraries ${ }^{137}$. Many peptides exert anticancer functions and F3 is known for mediating the delivery to specific tumour cells and tumour angiogenesis by binding to NCL expressed on the surface of tumour cells and endothelial cells as angiogenesis precursors ${ }^{138}$. Indeed, NCL facilitates the internalization of F3 peptide into tumour cells and transport into the cell nucleus. Therefore, macromolecular drugs conjugated with F3 peptides might be efficiently internalized into tumour cells via NCL-mediated transport, resulting in enhanced anti-cancer effects. Moreover, F3 contains a CendR sequence that enables deep penetration of the tumour parenchyma and promotes local drug aggregation and absorption ${ }^{139}$.

\subsection{F3 peptide-conjugated nanoparticles}

The F3-peptide has been investigated in recent years as it represents an important ligand for $\mathrm{NCL}$ to be used for a targeted therapy against NCL-overexpressing cancer cells ${ }^{140}$. Among the developed nano-formulations, F3-functionalized PEG-PLGA nanoparticles were proposed for the delivery of anticancer drugs, such as paclitaxel (PTX) in the treatment of glioma (Hu Q et al 2013). The aim was to increase the efficacy of chemotherapeutic drugs and to improve the ability of nanocarriers to pass the blood-brain barrier in order to reach the tumour site ${ }^{141}$, ${ }^{142}$. Moreover, the effectiveness of nanoparticles systems in the treatment of glioma is limited by the difficulty to extravasate from the blood vessels and invade the tumour parenchyma because of the small size of pores for penetration in glioma ${ }^{143,144}$. C-end Rule (CendR) peptides were demonstrated to be able to promote extravasation and tissue penetration through cell internalization after binding to their receptor neuropilin-1 (NRP1) on endothelial 
cells ${ }^{145,146}$. thyp-1 is a peptide containing a CendR motif and reported the ability to mediate tissue penetration through NRP1 ${ }^{147}$. Therefore, the co-administration of tLyp-1 and F3functionalized nanoparticles was investigated as it may constitute an efficient system to mediate a specific target therapy for glioma and to improve cellular internalization and extravasation. F3 was linked to NPs through a maleimide-thiol coupling reaction and the size of the formulation were around $150 \mathrm{~nm}$. In vitro release results showed a faster initial release of PTX followed by a mild and controlled release 10 hours after the administration. A high accumulation of F3-NPs was found in C6 glioma cell lines, confirming the ability of F3 to promote the binding of the NPs to the surface of C6 cells. PTX loaded F3-NPs revealed an enhanced anti-proliferative effect on C6 cells compared to F3-free NPs. 3D multicellular tumour spheroids were used as a model for evaluating the infiltration ability of F3-NPs, which showed to penetrate deeper than unmodified NPs. In vivo glioma distribution experiment was performed on Balb/c nude mice xenografted with $\mathrm{C} 6$ cells using a non-invasive fluorescence imaging system which revealed a maximum fluorescent intensity of NPs at $12 \mathrm{~h}$ followed by a decrease $24 \mathrm{~h}$ post-injection. NPs co-administered with thyp-1 displayed a higher fluorescence at the glioma site. Furthermore, the co-administration of F3-NPs and tLyp-1 allowed a higher accumulation at the tumour site. Mice treated with F3-Nps and tLyp-1 prolonged survival presented a significant prolonged survival (median survival of 42 days) and this improved antiglioma effect can be explained by the enhanced accumulation of PTX at the glioma region resulting from the F-3 decoration and tLyp-1 peptide co-administration.

A theranostic approach based on NPs was developed by Li $Y$ et al ( $L i$ 2019) using sonodynamic therapy (SDT) which has been investigated as a reactive oxygen species (ROS)mediated cancer therapy and high-intensity focused ultrasound (HIFU). HIFU ablation provides a promising non-invasive strategy for the therapy of solid tumours ${ }^{148}$. The advantages of SDT include the high efficacy in penetrating the tumour tissue in a specific manner thus minimizing the damage of normal tissues ${ }^{149}$. However, SDT presents some limits such as a high cavitation threshold, low quantum yield of the sonosensitizer and also nonthermal ablation effects which lead to toxic free radicals and singlet oxygen generation during the HIFU process ${ }^{150,151 .}$ Methylene blue (MB) can work as sonosensitizer for SDT but presents some limitations 83, 152 . Therefore, NPs may represent a solution for reducing the oxidation of MB in the environment and a functionalization of NPs may improve the absorption by tumour cells ${ }^{153}$. In this work, F3-targeted PLGA-NPs were prepared for combining dual-modal imaging and SDT. Photoacoustic imaging together with molecular targeted contrast agents may improve cancer imaging, while MRI provides cellular and molecular information in cancer ${ }^{154}$. A nanosystem was designed to load both PA and MRI contrast agents and its activity was evaluated on MDAMB-231 breast cancer cells and HUVECs. F3-PLGA@MB/Gd NPs were successfully prepared 
and characterized reporting a smooth, uniform and spherical morphology. MDA-MB-231 displayed the highest fluorescence after incubation with F3-NPs with a quick release after 5 min incubation followed by a gradual enhancement of intensity indicating the efficient targeting capacity of F3-NPs. 3D multicellular tumour spheroids were employed for reproducing the solid tumour tissue. Laser confocal images showed high fluorescence intensity in F3-targeted group compared to the non-targeted group, indicating that F3-functionalized NPs can be recognized and then internalized in the inner region of solid tumours. To induce the ROS generation by F3-PLGA@MB/Gd NPs, these NPs were incubated with RNO and histidine and treated with US. After the exposure time, ROS were generated thus revealing the capacity to kill tumour cells in SDT. The decoration of NPs with F3 allowed a higher accumulation of NPs in tumour cells leading to an increased ROS yield after incubation with US. Cell viability was investigated on MDA-MB-231 breast cancer cells and it remained high at all time points and concentrations assessing the cyto-safety of F3-PLGA@MB/Gd NPs. Cell death was not observed in the group of cells without US, while the F3-NPs displayed the lowest cell survival rate. An in vivo imaging system was used to evaluate the biodistribution of the formulation in BALB/c nude mice injected with MDA-MB-231 cells and reported an increased fluorescence in the F3-targeted revealing a high accumulation of the NPs in the target tissue. The tumour to background ration (TBR) allowed measuring the fluorescence intensity ratio and was reported higher in the targeted group. PA/MRI of the F3-NPs were investigated, and the PA signal intensity increased with different concentrations using $710 \mathrm{~nm}$ for excitation. Highresolution $\mathrm{PA}$ and $\mathrm{MRI}$ imaging of the tumour tissue could be performed in the F3PLGA@MB/Gd NPs group and the tumour signals were concentrated at 6h post-injection. Therefore, F3-PLGA@MB/Gd reported a potential use as PA and MRI contrast agent, thus helping in image-guided therapies. Concerning HIFU, the quantitative coagulation necrosis volume in PLGA@MB/Gd + HIFU group was 9 times larger than the HIFU group, indicating that PLGA@MB/Gd NPs can be employed as synergistic ablative agent in HIFU therapy. The effect of HIFU together with F3-PLGA@MB/Gd NPs was assessed with xenograft tumours in nude mice and, after HIFU irritation, the mean gray scale difference in the F3-PLGA@MB/Gd NPs group was higher than PBS group and non-targeted group. Moreover, the combination of F3-PLGA@MB/Gd NPs and HIFU led to an increased efficiency of tumour ablation and the induction of tumour cell apoptosis in surrounding areas. Even in further studies on ROS need to be performed during the HIFU irradiation in vivo, the results of this work provide evidence of the fact that the combination of HIFU ablation and SDT by using F3-PLGA@MB/Gd as a sonosensitizer allowed to killing the residual live tumour cells.

Another study presented the F3-conjugated polyacrylamide (PAA) NPs for the treatment of prostate cancer (PCa) assisted by photoacoustic imaging (PAI) ${ }^{155}$. Polymer based hydrogel NPs are biocompatible and chemically inert with a long circulation life and a reduced toxicity 
of the functional loadings ${ }^{156,157}$. PAl is a novel imaging modality important in prostate imaging combining an excellent optical sensitivity for observing macroscopic, chemical contents and US resolution for evaluating physical architectures in the tissues ${ }^{158,159}$. Confocal microscopy images revealed that the functionalization with $\mathrm{F} 3$ peptide improved the uptake of PEG-PAA NPs into DU145 PCa cells. For the in vivo study, subcutaneous DU145 xenograft tumours were induced in nude rats and, once the tumours formed, the rats were injected with F3conjugated PEG-PAA NPs and PAI was performed. The tumours could be identified also in the PA imaging on a canine prostate. F3-conjugated NPs were able to stain the microarchitectures in transgenic mice. They developed a tumour pathologically similar to the human PCa and were treated with NPs. 4 hours after the treatment, H\&E on the prostate samples revealed representative microarchitecture patterns. Therefore, F3-functionalized hydrogels NPs can improve the optical contrast of PCa tumours and allow observing the tumour tissue architectures.

\section{N6L as a ligand for NCL}

For several years, our research team has developed ligands of peptide origin of NCL belonging to a family of molecules called Nucant for NUCleolin ANTagonist. These multivalent pseudopeptides are composed of a polypeptide matrix rich in lysine residues on which are covalently linked several Lys [CH2N] Pro-Arg pseudotripeptides and inducing apoptosis of tumor cells [15]. In order to improve the specific activity of these molecules, several analogues have been synthesized by varying the size and type of matrix as well as the number of pseudotripeptides grafted onto it. Thus, the effects of these compounds on tumour growth were tested and comparative studies indicated that the optimal inhibitory effect was obtained with a molecule composed of 6 pseudotripeptides called N6L (ref). N6L interacts with the carboxyterminal RGG domain of NCL inhibiting the binding of several growth factors involved in cell growth such as midkine [93], pleiotrophin [94] and also lactoferin [54]. Since NCL is overexpressed on the surface of tumour cells, we have shown that N6L not only has the property of inducing apoptosis in tumour cells but also of being able to target tumour tissues 46, 160 leading us to use this pseudopeptide to functionalize nanoparticles for therapeutic purposes against cancer.

\subsection{N6L-functionalized nanocapsules}

Among the different approaches for synthesizing nanovectors, the design of nanometre-sized hollow polymeric particles, also called nanocapsules, has received increasing interest as it allows accessing to a wide range of nanovector presenting tuneable diameters, wall thickness, mechanical property and permeability. 161,162 Different strategies have been reported to prepare nanocapsules 163 including emulsion-diffusion, emulsion-coacervation, double 
emulsification, nanoprecipitation, combining polymer-coating and layer-by-layer process. In this review, we only focus on capsules obtained through the multilayer deposition of polyelectrolytes via the layer-by-layer (LbL) process on a sacrificial template ${ }^{164}$, i.e. "template method" ${ }^{165-167}$ (figure 2). At this stage of preparation, the template can be removed to yield nanocapsule in which other therapeutic molecules can be included. This method of preparation allows not only creating capsules, but also allows to easily control the particle size and shape, the surface functionality and the shell permeability and thickness.

Indeed, the LbL assembly driven by electrostatic interactions allows elaborating multilayer polyelectrolyte films with well-controlled composition, thickness and chemical structure, and those simply by alternating the deposition of oppositely charged polymers (i.e. polyelectrolyte) onto a charged substrate (figure 3). ${ }^{168}$ This approach is achieved in aqueous solutions and is well suited for the fabrication of homogeneous and ultrathin films leading to efficient solutions for numerous applications as biomedical ones.

Several physicochemical parameters (e.g. adsorption time, charge density, $\mathrm{pH}$, temperature, ionic strength, average molar mass...) have been evidenced to strongly impact the final characteristics of polyelectrolyte multilayer assemblies. One may cite the thickness, permeability, roughness, wettability or even swelling behaviour. ${ }^{169-174}$ For a more in-depth discussion on the LbL process, the reader is kindly invited to have a look on an excellent recent review by Borges and Mano. ${ }^{175}$

Applied to fabricate multifunctional nanocapsules, the strategy consists of a successive deposition of polyelectrolyte layers on a surface of a given template leading to a high structural control. ${ }^{165,167,176,177}$ Then, the selective removal of the templates induces the obtention of the capsule. Thus, this is a simple and a straightforward approach which could be applied to a wide range of materials, including organic or inorganic templates as calcium carbonate particles ${ }^{178-180}$ vesicle/liposome ${ }^{181}$ liquid template ${ }^{182-186}$ metal oxide nanoparticles ${ }^{187}$ or gold nanoparticles as templates. ${ }^{188-193}$

Recently, we have investigated the elaboration of nanocapsules based on N6L ${ }^{194}$ which exhibit anticancer activity by using gold nanoparticles as templates (figure 4). We judiciously take advantage of the electrostatic interactions of the pseudopeptide N6L which is a polycation (18 positive charges at physiological $\mathrm{pH}$ ) to construct the multilayer films. We evidence the possibility to control (i) the amount of loaded N6L through controlling the size of the gold template (studied diameter: $20 \mathrm{~nm}$ and $60 \mathrm{~nm}$ ) (ii) the resulting mechanical property of the N6L-based particles and (iii) the chemical structure of the last layer constituting the particle (N6L is known to allow a specific targeting of cancer cells, then we have selected the N6L as last layer).

We have selected gold nanoparticles as template as it combines very interesting properties. Indeed, these nanomaterials (i) are commercially available in a large range of size as of $5 \mathrm{~nm}$, 
(ii) are biocompatible and (iii) present an excellent contrast for electron microscopy study. The deposition of successive polyelectrolyte multilayers onto gold nanoparticles ${ }^{195,196}$ have been followed by zeta potential measurement and by exploiting the sensitivity of the gold nanoparticle's surface Plasmon band due to the adsorbed species. ${ }^{197}$ With this last technique, we also prove that there is no aggregation during the adsorption of the polyelectrolyte layer including the N6L on the colloidal surface.

Dissolving the sacrificial gold core leads to the formation of N6L-based nanocapsules. For this, we have used the potassium cyanide which is the most used chemical for the gold hydrolysis. Indeed, the cyanide-assisted etching induces the formation of a water-soluble salt of potassium gold cyanide complex (see equation 1).

$$
4 \mathrm{Au}+8 \mathrm{KCN}+2 \mathrm{H}_{2} \mathrm{O}+\mathrm{O}_{2} \rightarrow 4 \mathrm{KOH}+4 \mathrm{KAu}(\mathrm{CN})_{2} \quad \text { Eq. } 1
$$

The $\mathrm{KAu}(\mathrm{CN})_{2}$ complex is highly toxic but it can easily be eliminated through diffusion across the polyelectrolyte membrane. ${ }^{189,198}$ This process of core dissolution has been followed by UV spectrophotometric monitoring of the disappearance of the gold Plasmon absorbance band. Transmission electron microscopy (TEM) has been used to evidence the formation of the capsule.

Using this procedure, we obtained functionalized $20 \mathrm{~nm}$ and $60 \mathrm{~nm}$ nanocapsules with N6L. As shown in figure 5, the $20 \mathrm{~nm}$ and $60 \mathrm{~nm}$ functionalized nanocapsules inhibited in dosedependent manner the survival of tumour cells derived from human. It is noteworthy that the specific activity of the nanocapsules is dependent on their size. While an equivalent of 6 $\mu \mathrm{M}$ N6L linked to the $20 \mathrm{~nm}$ nanocapsules induced in $72 \mathrm{~h}, 87 \pm 0.58 \%$ of cell death, same equivalent of $\mathrm{N} 6 \mathrm{~L}$ linked to the $60 \mathrm{~nm}$ size nanocapsules, induced $65 \pm 2.5 \%$ of cell death. The reasons for this difference remain unclear but are currently investigated.

In addition, in recent study we demonstrated that these nanostructures target cancer cells derived from pancreatic cancer suggesting that this material could be used as theranostic tool. Studies are currently in progress to evaluate this possibility to use them for in vivo studies. Compared to commercialized particles, these novel systems obtained by combining template method and the LbL process (N6L is implied as polyelectrolyte during this process), present the benefits to lead a well-defined carrier and also to specifically target tumour cells (nanoparticle concentration, size, N6L loaded)

\subsection{Hollow mesoporous silica microspheres as starting material for fabrication of N6L- based hollow particles}

The use of hollow mesoporous silica microspheres (HMSM) as starting material is an appealing alternative because it can easily be synthesized in controlled manner and it is a non-toxic material at low concentration. Mesoporous silica particles have a porous structure that can be exploited to load chemicals of interest with a high loading capacity. Moreover, the surface can 
easily be chemically modified. In this context, we have recently reported on the possibility to use hollow mesoporous silica microspheres as N6L carriers for pancreatic cancer treatment and on the controlled $\mathrm{N} 6 \mathrm{~L}$ release according to the $\mathrm{pH}$ media (i.e. release in acidic condition). 199 The hollow mesoporous silica microspheres characteristic allow controlling the resulting size and N6L loading capacity. The $\mathrm{pH}$ response has been targeted as it is an interesting stimulus for pancreatic cancer treatment. Indeed, the $\mathrm{pH}$ value of the extracellular medium in pancreatic tumours is lower than that of healthy tissue. We then propose to use the chitosan which is a biopolymer as a pH-sensitive gatekeeper (chitosan's pKa 6.2-7.0). This polysaccharide was grafted to the hollow mesoporous silica microspheres surface (figure 6) and then the particle was loaded with the pro-apoptotic NCL antagonist agent (N6L) as an anticancer drug (figure 7).

The obtained particles allow controlling the N6L loading according to the porosity parameter of the HMSM particles, i.e. $44 \%$ of N6L loading for a particle having this characteristic: 116 $\mathrm{m}^{2} \cdot \mathrm{g}^{-1}$, a total pore volume $0.64 \mathrm{~cm}^{3} \cdot \mathrm{g}^{-1}$ and a size of $623 \mathrm{~nm}(\mathrm{PDI}=0.40)$ and $25 \%$ of $\mathrm{N} 6 \mathrm{~L}$ loading for a HMSM having the following characteristic: $206 \mathrm{~m}^{2} \cdot \mathrm{g}^{-1}$, a total pore volume $0.72 \mathrm{~cm}^{3} \cdot \mathrm{g}^{-1}$ and a size of $530 \mathrm{~nm}(\mathrm{PDI}=0.30)$.

The obtained N6L-based chitosan-hollow mesoporous silica microspheres present $\mathrm{pH}$ responsive drug releases and excellent anticancer activities with inhibition of cancer cell growth, i.e. high killing potency for the diseased cells. To evidence this, blue Alamar assays have been used for evaluating the cell viability submitted to different concentrations of N6Lloaded chitosan-HMSM particles (1.5 to $100 \mu \mathrm{M})$. The inhibition of cancer cell growth was $48 \%$ and $60 \%$ according to the characteristic of the chitosan-hollow mesoporous silica microspheres. This new study ${ }^{199}$ demonstrates a new method for the controlled loading and release of $\mathrm{N} 6 \mathrm{~L}$, distinct from other systems such as iron oxide magnetic nanoparticles with the N6L ${ }^{200}$ or N6L-polyplexes. ${ }^{201}$

\subsection{N6L-functionalized magnetic nanoparticles}

Within this broad spectrum of nanomaterials, magnetic nanoparticles (NPM) have, in recent years, gained special attention thanks to their intrinsic magnetic property and their potential to produce a rise in temperature. NPM are formed from a metallic body embedded in an organic polymer or an inorganic shell. There are several types of NPMs such as those based on manganese, cobalt, nickel and also their derivatives in the form of oxides. While all of these metals are excellent contrast agents for MRI scans, some can exhibit tissue toxicity. Currently, iron oxide NPM remains the subject of much attention. Introduced in the clinic more than twenty years ago, their use is frequent as a contrast agent in MRI. These NPM have been shown to be well tolerated, biocompatible and free from toxicity. For several years, these NPM have aroused the interest of much research and their use has extended to other medical applications 
which will be discussed below 202-206. Regarding their design, several physicochemical parameters are required for the synthesis of NPM. These parameters depend on the medical application for which these NPMs are intended. For diagnosis in MRI and treatment by magnetic hyperthermia ( $\mathrm{MH})$ in oncology, the parameters to be taken into consideration are the following: the composition of the core and the surface, the hydrodynamic diameter, the functionalization and the heating power or "Specifie Absorption Rate" (SAR) ${ }^{207-209}$. They are formed of an iron oxide core coated in an organic or inorganic matrix. The nucleus consists of particles of maghemite $\left(\mathrm{Fe}_{2} \mathrm{O}_{3}\right)$ or magnetite $\left(\mathrm{Fe}_{3} \mathrm{O}_{4}\right){ }^{210-213}$. The iron core is covered by a polymer coating consisting of a layer of polymers whose roles are to prevent the agglomeration of iron oxide, to limit non-specific interactions with the cell, to facilitate conjugation at the surface of targeting agents and drugs and significantly modulate the pharmacokinetics of NPMs. The most commonly used coatings are polyethylene glycol (PEG), dextran, chitosan, phospholipids and dimercaptosuccinic acid (DMSA) ${ }^{213}$.

NPM are referred to as SPION (Super Paramagnetic Iron Oxide Nanoparticles) when their hydrodynamic diameter is between $50 \mathrm{~nm}$ and $150 \mathrm{~nm}$, or USPION (Ultrasmall Super Paramagnetic Iron Oxide Nanoparticles) when the hydrodynamic diameter is less than $50 \mathrm{~nm}$ 214, 215. Paramagnetism designates in magnetism the behaviour of a material which does not have spontaneous magnetization but which, under the effect of an external magnetic field, acquires a magnetization directed in the same direction under the effect of a magnetic field exterior. At the nanometric scale, the magnetic energy is about $10^{4}$ times higher, giving a superparamagnetic property ${ }^{216}$.

Among the important parameters, the surface charge and the hydrophobicity can affect the biodistribution of nanoparticles by limiting or promoting interactions with plasma proteins, those of the extracellular matrix and non-target cells. Charged nanoparticles and hydrophobic nanoparticles have a short lifespan in circulation due to adsorption to plasma proteins (opsonization) followed by their elimination. Positively charged nanoparticles can bind nonspecifically to non-target cells leading to non-specific internalization. The hydrophobic groups present on the surface of the nanoparticles cause agglomeration during the injection leading to a rapid elimination by the reticuloendothelial system (macrophages and monocytes). Modifying the surface with hydrophilic molecules such as PEG makes it possible to reduce the phenomenon of opsonization and thus prolongs the circulation time of nanoparticles $206,213,217$. In order to give them a specificity of action, these NPMs can be functionalized by covalent bond strategies or by physical interactions. The first one consists in directly binding the molecule to the surface of the nanoparticle carrying a chemical group, or indirectly by the intermediary of an additional chemical group, always by covalent bonds. Physical interactions group together electrostatic interactions and hydrophilic/hydrophobic interactions. These bonds are less stable than covalent bonds, but their advantage lies in the rapidity of their 
constitution and the unnecessary need for intermediate stages of chemical modifications. The choice of strategy obviously depends on the chemical properties of the molecule to be grafted and on the functional groups present at the surface. It is especially important to stress that whatever the functionalization strategy, it must preserve the functionality of the molecules grafted to the nanoparticle ${ }^{213}$. Functionalization of MNPs represents a great interest related to their clinical applications.

In this context, the pseudopeptide N6L has been used to functionalize iron oxide nanoparticles combining magnetic hyperthermia and anti-cancer drug delivery in the treatment of breast cancer xenografts ${ }^{200}$. In this study, MNPs were electrostatically functionalized with N6L (N6LMNP), doxorubicin (DOX-MNP) or both (N6L-DOX-MNP) as described by Kossatz et al., ${ }^{218}$ and their antitumoral effect has been evaluated in vitro as well as in vivo using breast adenocarcinoma cell lines MDA-MB-231. In this study, we showed that the presence of N6L linked to MNP, improved the intracellular uptake of MNP in tumour cells. This data has been validated by in vivo data in which we showed intraperitoneal injection of N6L-DOX-MNP in mice with xenografted tumours, led to significant reduction in tumour volume. These data highlight that the presence of the pseudopeptide N6L on the MNP surface might well be beneficial in its function as an enhancer of MNP internalization into cancer cells opening the possibility of the induction of intracellular heating spots and, in the end, tumour cell death. This potential has been demonstrated in a recent study showing that treatment with N6L-MNP combined with chemotherapeutic drugs, causes, in association with hyperthermia, a tumour regression in pancreatic xenograft model ${ }^{219}$.

Using this approach, we find that the therapeutic effect of magnetic hyperthermia is strongly enhanced by the association of N6L linked to the leading to tumour regression ${ }^{219}$.

\section{Conclusions and oulook}

This review focuses on the anti-tumour properties of nanoparticles functionalized with NCL ligands as a delivery agent for therapeutic molecules for cancer therapy and diagnosis.

Over-expressed in almost all types of cancer cells, studies on NCL have been increasing over the last ten years as a molecule of interest and therapeutic target in cancer.

Thus, the NCL ligands such as the aptamer AS1411 and the F3 and N6L peptides which are the subject of this review have been selected because of their specific tumour targeting properties associated with anti-tumour effects in the case of AS1411 and N6L. These two molecules have been studied in human clinical trials without any adverse effects being observed at therapeutic doses.

Moreover, grafted onto different types of nanoparticles, the anti-tumour effect of these tools has been demonstrated in numerous experimental models of different types of cancer. Interestingly, we have shown that it is possible to obtain nanoparticles functionalized with 
chemotherapeutic agents, targeting the tumour tissue and capable of inducing local heating, inhibiting tumour growth. Referring to the literature, it is now clear that the solution for irradiating a pathology will involve blocking and inhibiting several pathways that are activated in the process leading to tumour growth. It is therefore clear that the use of nanoparticle-type platforms as described in this review combining tumour targeting, chemotherapy and thermotherapy represent a promising therapeutic possibility against cancer or even other pathologies where nucleolin could be involved.

\section{Conflicts of interest}

There are no conflicts to declare.

\section{Acknowledgments}

This research was supported by the Agence Nationae de la Recherche I (ANR), GLYCONUC project 16-CE17-0023, the "Ligue contre le cancer" and with the financial support from Inserm Cancer (PANTHER project). 


\section{References}

1. Tran, S.; DeGiovanni, P. J.; Piel, B.; Rai, P., Cancer nanomedicine: a review of recent success in drug delivery. Clin Transl Med 2017, 6 (1), 44.

2. Baudino, T. A., Targeted Cancer Therapy: The Next Generation of Cancer Treatment. Curr Drug Discov Technol 2015, 12 (1), 3-20.

3. Schirrmacher, V., From chemotherapy to biological therapy: A review of novel concepts to reduce the side effects of systemic cancer treatment (Review). Int J Oncol 2019, 54 (2), 407-419.

4. Bahrami, B.; Hojjat-Farsangi, M.; Mohammadi, H.; Anvari, E.; Ghalamfarsa, G.; Yousefi, M.; Jadidi-Niaragh, F., Nanoparticles and targeted drug delivery in cancer therapy. Immunology Letters 2017, 190, 64-83.

5. Brigger, I.; Dubernet, C.; Couvreur, P., Nanoparticles in cancer therapy and diagnosis. Advanced drug delivery reviews 2012, 64, 24-36.

6. Davis, M. E.; Chen, Z.; Shin, D. M., Nanoparticle therapeutics: an emerging treatment modality for cancer. In Nanoscience and technology: A collection of reviews from nature journals, World Scientific: 2010; pp 239-250.

7. Jain, R. K.; Stylianopoulos, T., Delivering nanomedicine to solid tumors. Nature reviews Clinical oncology 2010, 7 (11), 653.

8. Petros, R. A.; DeSimone, J. M., Strategies in the design of nanoparticles for therapeutic applications. Nature reviews Drug discovery 2010, 9 (8), 615-627.

9. Sharma, A.; Goyal, A. K.; Rath, G., Recent advances in metal nanoparticles in cancer therapy. Journal of drug targeting 2018, 26 (8), 617-632.

10. Liu, C. C.; Yang, H.; Zhang, R.; Zhao, J. J.; Hao, D. J., Tumour-associated antigens and their anti-cancer applications. European journal of cancer care 2017, 26 (5), e12446.

11. Menard, S.; Pupa, S. M.; Campiglio, M.; Tagliabue, E., Biologic and therapeutic role of HER2 in cancer. Oncogene 2003, 22 (42), 6570-6578.

12. Dong, Y.; Li, W.; Gu, Z.; Xing, R.; Ma, Y.; Zhang, Q.; Liu, Z., Inhibition of HER2-Positive Breast Cancer Growth by Blocking the HER2 Signaling Pathway with HER2-Glycan-Imprinted Nanoparticles. Angewandte Chemie International Edition 2019, 58 (31), 10621-10625.

13. Daver, N.; Schlenk, R. F.; Russell, N. H.; Levis, M. J., Targeting FLT3 mutations in AML: review of current knowledge and evidence. Leukemia 2019, 33 (2), 299-312.

14. Vyse, S.; Huang, P. H., Targeting EGFR exon 20 insertion mutations in non-small cell lung cancer. Signal transduction and targeted therapy 2019, 4 (1), 1-10.

15. Tian, T.; Li, X.; Zhang, J., mTOR signaling in cancer and mTOR inhibitors in solid tumor targeting therapy. International journal of molecular sciences 2019, 20 (3), 755.

16. Katsuno, Y.; Meyer, D. S.; Zhang, Z.; Shokat, K. M.; Akhurst, R. J.; Miyazono, K.; Derynck, R., Chronic TGF- $\beta$ exposure drives stabilized EMT, tumor stemness, and cancer drug resistance with vulnerability to bitopic mTOR inhibition. Science signaling 2019, 12 (570), eaau8544.

17. Zhen, X.; Cheng, P.; Pu, K., Recent advances in cell membrane-camouflaged nanoparticles for cancer phototherapy. Small 2019, 15 (1), 1804105.

18. Sau, S.; Alsaab, H. O.; Bhise, K.; Alzhrani, R.; Nabil, G.; lyer, A. K., Multifunctional nanoparticles for cancer immunotherapy: A groundbreaking approach for reprogramming malfunctioned tumor environment. Journal of Controlled Release 2018, 274, 24-34.

19. Miller, K. D.; Nogueira, L.; Mariotto, A. B.; Rowland, J. H.; Yabroff, K. R.; Alfano, C. M.; Jemal, A.; Kramer, J. L.; Siegel, R. L., Cancer treatment and survivorship statistics, 2019. CA Cancer J Clin 2019, 69 (5), 363-385. 
20. Seto, M.; Honma, K.; Nakagawa, M., Diversity of genome profiles in malignant lymphoma. Cancer Sci 2010, 101 (3), 573-8.

21. de Martel, C.; Georges, D.; Bray, F.; Ferlay, J.; Clifford, G. M., Global burden of cancer attributable to infections in 2018: a worldwide incidence analysis. Lancet Glob Health 2020, 8 (2), e180-e190.

22. Collaborators, G. B. D. R. F., Global burden of 87 risk factors in 204 countries and territories, 1990-2019: a systematic analysis for the Global Burden of Disease Study 2019. Lancet 2020, 396 (10258), 1223-1249.

23. Dagogo-Jack, I.; Shaw, A. T., Tumour heterogeneity and resistance to cancer therapies. Nat Rev Clin Oncol 2018, 15 (2), 81-94.

24. Norouzi, M.; Amerian, M.; Amerian, M.; Atyabi, F., Clinical applications of nanomedicine in cancer therapy. Drug Discov Today 2020, 25 (1), 107-125.

25. Wolfram, J.; Ferrari, M., Clinical Cancer Nanomedicine. Nano Today 2019, 25, 85-98.

26. Park, J.; Choi, Y.; Chang, H.; Um, W.; Ryu, J. H.; Kwon, I. C., Alliance with EPR Effect: Combined Strategies to Improve the EPR Effect in the Tumor Microenvironment. Theranostics 2019, 9 (26), 8073-8090.

27. Shi, Y.; van der Meel, R.; Chen, X.; Lammers, T., The EPR effect and beyond: Strategies to improve tumor targeting and cancer nanomedicine treatment efficacy. Theranostics 2020, 10 (17), 7921-7924.

28. Vert, M.; Doi, Y.; Hellwich, K.-H.; Hess, M.; Hodge, P.; Kubisa, P.; Rinaudo, M.; Schué, F., Terminology for biorelated polymers and applications (IUPAC Recommendations 2012). Pure and Applied Chemistry 2012, 84 (2), 377-410.

29. Bazak, R.; Houri, M.; El Achy, S.; Kamel, S.; Refaat, T., Cancer active targeting by nanoparticles: a comprehensive review of literature. J Cancer Res Clin Oncol 2015, 141 (5), 769-84.

30. Pucci, C.; Martinelli, C.; Ciofani, G., Innovative approaches for cancer treatment: current perspectives and new challenges. Ecancermedicalscience 2019, 13, 961.

31. Das, S. S.; Bharadwaj, P.; Bilal, M.; Barani, M.; Rahdar, A.; Taboada, P.; Bungau, S.; Kyzas, G. Z., Stimuli-Responsive Polymeric Nanocarriers for Drug Delivery, Imaging, and Theragnosis. Polymers (Basel) 2020, 12 (6).

32. Ventola, C. L., Progress in nanomedicine: approved and investigational nanodrugs. Pharmacy and Therapeutics 2017, 42 (12), 742.

33. Qi, J.; Li, H.; Liu, N.; Xing, Y.; Zhou, G.; Wu, Y.; Liu, Y.; Chen, W.; Yue, J.; Han, B., The implications and mechanisms of the extra-nuclear nucleolin in the esophageal squamous cell carcinomas. Medical Oncology 2015, 32 (3), 45.

34. Scott, D. D.; Oeffinger, M., Nucleolin and nucleophosmin: nucleolar proteins with multiple functions in DNA repair. Biochem Cell Biol 2016, 94 (5), 419-432.

35. Chen, Z.; Xu, X., Roles of nucleolin. Focus on cancer and anti-cancer therapy. Saudi Med J 2016, 37 (12), 1312-1318.

36. Ma, N.; Matsunaga, S.; Takata, H.; Ono-Maniwa, R.; Uchiyama, S.; Fukui, K., Nucleolin functions in nucleolus formation and chromosome congression. J Cell Sci 2007, 120 (Pt 12), 2091-105.

37. Ginisty, H.; Sicard, H.; Roger, B.; Bouvet, P., Structure and functions of nucleolin. J Cell Sci 1999, 112 ( Pt 6), 761-72.

38. Caizergues-Ferrer, M.; Belenguer, P.; Lapeyre, B.; Amalric, F.; Wallace, M. O.; Olson, M. O., Phosphorylation of nucleolin by a nucleolar type NII protein kinase. Biochemistry 1987, 26 (24), 7876-83. 
39. Abdelmohsen, K.; Gorospe, M., RNA-binding protein nucleolin in disease. RNA Biol 2012, 9 (6), 799-808.

40. Lischwe, M. A.; Cook, R. G.; Ahn, Y. S.; Yeoman, L. C.; Busch, H., Clustering of glycine and NG,NG-dimethylarginine in nucleolar protein C23. Biochemistry 1985, 24 (22), 6025-8.

41. Hovanessian, A. G.; Puvion-Dutilleul, F.; Nisole, S.; Svab, J.; Perret, E.; Deng, J. S.; Krust, B., The cell-surface-expressed nucleolin is associated with the actin cytoskeleton. Exp Cell Res 2000, 261 (2), 312-28.

42. Srivastava, M.; Pollard, H. B., Molecular dissection of nucleolin's role in growth and cell proliferation: new insights. FASEB J 1999, 13 (14), 1911-22.

43. Ugrinova, I.; Monier, K.; Ivaldi, C.; Thiry, M.; Storck, S.; Mongelard, F.; Bouvet, P., Inactivation of nucleolin leads to nucleolar disruption, cell cycle arrest and defects in centrosome duplication. BMC Mol Biol 2007, 8, 66.

44. Krust, B.; El Khoury, D.; Nondier, I.; Soundaramourty, C.; Hovanessian, A. G., Targeting surface nucleolin with multivalent HB-19 and related Nucant pseudopeptides results in distinct inhibitory mechanisms depending on the malignant tumor cell type. BMC Cancer 2011, $11,333$.

45. Morfoisse, F.; Tatin, F.; Hantelys, F.; Adoue, A.; Helfer, A. C.; Cassant-Sourdy, S.; Pujol, F.; Gomez-Brouchet, A.; Ligat, L.; Lopez, F.; Pyronnet, S.; Courty, J.; GuillermetGuibert, J.; Marzi, S.; Schneider, R. J.; Prats, A. C.; Garmy-Susini, B. H., Nucleolin Promotes Heat Shock-Associated Translation of VEGF-D to Promote Tumor Lymphangiogenesis. Cancer Res 2016, 76 (15), 4394-405.

46. Destouches, D.; El Khoury, D.; Hamma-Kourbali, Y.; Krust, B.; Albanese, P.; Katsoris, P.; Guichard, G.; Briand, J. P.; Courty, J.; Hovanessian, A. G., Suppression of tumor growth and angiogenesis by a specific antagonist of the cell-surface expressed nucleolin. PloS one 2008, 3 (6), e2518.

47. El Khoury, D.; Destouches, D.; Lengagne, R.; Krust, B.; Hamma-Kourbali, Y.; Garcette, M.; Niro, S.; Kato, M.; Briand, J.-P.; Courty, J., Targeting surface nucleolin with a multivalent pseudopeptide delays development of spontaneous melanoma in RET transgenic mice. $B M C$ cancer 2010, 10 (1), 325.

48. Gilles, M.-E.; Maione, F.; Cossutta, M.; Carpentier, G.; Caruana, L.; Di Maria, S.; Houppe, C.; Destouches, D.; Shchors, K.; Prochasson, C., Nucleolin targeting impairs the progression of pancreatic cancer and promotes the normalization of tumor vasculature. Cancer research 2016, 76 (24), 7181-7193.

49. Jain, N.; Zhu, H.; Khashab, T.; Ye, Q.; George, B.; Mathur, R.; Singh, R. K.; Berkova, Z.; Wise, J. F.; Braun, F. K., Targeting nucleolin for better survival in diffuse large B-cell lymphoma. Leukemia 2018, 32 (3), 663-674.

50. Kashat, L.; So, A. K.-C.; Masui, O.; Wang, X. S.; Cao, J.; Meng, X.; MacMillan, C.; Ailles, L. E.; Siu, K. M.; Ralhan, R., Secretome-based identification and characterization of potential biomarkers in thyroid cancer. Journal of proteome research 2010, 9 (11), 5757-5769. 51. Li, L.; Hou, J.; Liu, X.; Guo, Y.; Wu, Y.; Zhang, L.; Yang, Z., Nucleolin-targeting liposomes guided by aptamer AS1411 for the delivery of siRNA for the treatment of malignant melanomas. Biomaterials 2014, 35 (12), 3840-3850.

52. Meng, G.-Z.; Xiao, S.-J.; Zeng, S.-E.; Li, Y.-Q., Downregulation of cell-surface-expressed nucleolin inhibits the growth of hepatocellular carcinoma cells in vitro. Zhonghua zhong liu za zhi [Chinese journal of oncology] 2011, 33 (1), 23-27. 
53. Shin, S.-H.; Lee, G. Y.; Lee, M.; Kang, J.; Shin, H.-W.; Chun, Y.-S.; Park, J.-W., Aberrant expression of CITED2 promotes prostate cancer metastasis by activating the nucleolin-AKT pathway. Nature communications 2018, 9 (1), 1-14.

54. Watanabe, T.; Hirano, K.; Takahashi, A.; Yamaguchi, K.; Beppu, M.; Fujiki, H.; Suganuma, M., Nucleolin on the cell surface as a new molecular target for gastric cancer treatment. Biological and Pharmaceutical Bulletin 2010, 33 (5), 796-803.

55. Wolfson, E.; Goldenberg, M.; Solomon, S.; Frishberg, A.; Pinkas-Kramarski, R., Nucleolin-binding by ErbB2 enhances tumorigenicity of ErbB2-positive breast cancer. Oncotarget 2016, 7 (40), 65320.

56. Wu, R.; Li, L.; Bai, Y.; Yu, B.; Xie, C.; Wu, H.; Zhang, Y.; Huang, L.; Yan, Y.; Li, X., The long noncoding RNA LUCAT1 promotes colorectal cancer cell proliferation by antagonizing Nucleolin to regulate MYC expression. Cell death \& disease 2020, 11 (10), 1-13.

57. Xu, Z.; Joshi, N.; Agarwal, A.; Dahiya, S.; Bittner, P.; Smith, E.; Taylor, S.; PiwnicaWorms, D.; Weber, J.; Leonard, J. R., Knocking down nucleolin expression in gliomas inhibits tumor growth and induces cell cycle arrest. Journal of neuro-oncology 2012, 108 (1), 59-67.

58. Chen, Z.; Xu, X., Roles of nucleolin: Focus on cancer and anti-cancer therapy. Saudi medical journal 2016, 37 (12), 1312.

59. Sader, M.; Courty, J.; Destouches, D., Nanoparticles functionalized with ligands of cell surface nucleolin for cancer therapy and diagnosis. J Nanomed Nanotechnol 2015, 6 (310), 2. 60. Bywater, M. J.; Pearson, R. B.; McArthur, G. A.; Hannan, R. D., Dysregulation of the basal RNA polymerase transcription apparatus in cancer. Nature Reviews Cancer 2013, 13 (5), 299-314.

61. Berger, C. M.; Gaume, X.; Bouvet, P., The roles of nucleolin subcellular localization in cancer. Biochimie 2015, 113, 78-85.

62. Ishimaru, D.; Zuraw, L.; Ramalingam, S.; Sengupta, T. K.; Bandyopadhyay, S.; Reuben, A.; Fernandes, D. J.; Spicer, E. K., Mechanism of regulation of bcl-2 mRNA by nucleolin and A+ U-rich element-binding factor 1 (AUF1). Journal of Biological Chemistry 2010, 285 (35), 2718227191.

63. Huang, Y.; Shi, H.; Zhou, H.; Song, X.; Yuan, S.; Luo, Y., The angiogenic function of nucleolin is mediated by vascular endothelial growth factor and nonmuscle myosin. Blood 2006, 107 (9), 3564-3571.

64. Liang, P.; Jiang, B.; Lv, C.; Huang, X.; Sun, L.; Zhang, P.; Huang, X., The expression and proangiogenic effect of nucleolin during the recovery of heat-denatured HUVECs. Biochimica et Biophysica Acta (BBA)-General Subjects 2013, 1830 (10), 4500-4512.

65. Dai, C.; Lv, S.; Shi, R.; Ding, J.; Zhong, X.; Song, H.; Ma, X.; Fan, J.; Sun, B.; Wang, R., RETRACTED ARTICLE: Nuclear Protein C23 on the Cell Surface Plays an Important Role in Activation of CXCR4 Signaling in Glioblastoma. Molecular neurobiology 2015, 52 (3), 15211526.

66. Schall, T. J.; Proudfoot, A. E., Overcoming hurdles in developing successful drugs targeting chemokine receptors. Nature Reviews Immunology 2011, 11 (5), 355-363.

67. Xue, L. J.; Mao, X. B.; Ren, L. L.; Chu, X. Y., Inhibition of CXCL12/CXCR4 axis as a potential targeted therapy of advanced gastric carcinoma. Cancer medicine 2017, 6 (6), 14241436.

68. Xie, Q.; Guo, X.; Gu, J.; Zhang, L.; Jin, H.; Huang, H.; Li, J.; Huang, C., p85a promotes nucleolin transcription and subsequently enhances EGFR mRNA stability and EGF-induced malignant cellular transformation. Oncotarget 2016, 7 (13), 16636. 
69. Farin, K.; Schokoroy, S.; Haklai, R.; Cohen-Or, I.; Elad-Sfadia, G.; Reyes-Reyes, M. E.; Bates, P. J.; Cox, A. D.; Kloog, Y.; Pinkas-Kramarski, R., Oncogenic synergism between ErbB1, nucleolin, and mutant Ras. Cancer research 2011, 71 (6), 2140-2151.

70. Reister, S.; Mahotka, C.; van den Höfel, N.; Grinstein, E., Nucleolin promotes Wnt signaling in human hematopoietic stem/progenitor cells. Leukemia 2019, 33 (4), 1052-1054.

71. Luis, T. C.; Ichii, M.; Brugman, M. H.; Kincade, P.; Staal, F. J., Wnt signaling strength regulates normal hematopoiesis and its deregulation is involved in leukemia development. Leukemia 2012, 26 (3), 414-421.

72. McCubrey, J. A.; Steelman, L.; Bertrand, F. E.; Davis, N. M.; Abrams, S. L.; Montalto, G.; D'Assoro, A. B.; Libra, M.; Nicoletti, F.; Maestro, R., Multifaceted roles of GSK-3 and Wnt/ $\beta$-catenin in hematopoiesis and leukemogenesis: opportunities for therapeutic intervention. Leukemia 2014, 28 (1), 15-33.

73. Zylbersztejn, F.; Flores-Violante, M.; Voeltzel, T.; Nicolini, F.-E.; Lefort, S.; MaguerSatta, V., The BMP pathway: A unique tool to decode the origin and progression of leukemia. Experimental hematology 2018, 61, 36-44.

74. Koutsioumpa, M.; Papadimitriou, E., Cell surface nucleolin as a target for anti-cancer therapies. Recent patents on anti-cancer drug discovery 2014, 9 (2), 137-152.

75. Quiroz-Mercado, J.; Ramírez-Velázquez, N.; Partido, G.; Zenteno, E.; Chávez, R.; Agundis-Mata, C.; Jiménez-Martínez, M. C.; Garfias, Y., Tissue and cellular characterisation of nucleolin in a murine model of corneal angiogenesis. Graefe's Archive for Clinical and Experimental Ophthalmology 2016, 254 (9), 1753-1763.

76. Wang, Y.; Chen, X.; Tian, B.; Liu, J.; Yang, L.; Zeng, L.; Chen, T.; Hong, A.; Wang, X., Nucleolin-targeted extracellular vesicles as a versatile platform for biologics delivery to breast cancer. Theranostics 2017, 7 (5), 1360.

77. Zhang, H.; Ingham, E. S.; Gagnon, M. K. J.; Mahakian, L. M.; Liu, J.; Foiret, J. L.; Willmann, J. K.; Ferrara, K. W., In vitro characterization and in vivo ultrasound molecular imaging of nucleolin-targeted microbubbles. Biomaterials 2017, 118, 63-73.

78. Ansari, N.; Yazdian-Robati, R.; Shahdordizadeh, M.; Wang, Z.; Ghazvini, K., Aptasensors for quantitative detection of Salmonella Typhimurium. Anal Biochem 2017, 533, 18-25.

79. Zhang, Y.; Lai, B. S.; Juhas, M., Recent advances in aptamer discovery and applications. Molecules 2019, 24 (5), 941.

80. Bates, P. J.; Reyes-Reyes, E. M.; Malik, M. T.; Murphy, E. M.; O'toole, M. G.; Trent, J. O., G-quadruplex oligonucleotide AS1411 as a cancer-targeting agent: Uses and mechanisms. Biochimica et Biophysica Acta (BBA)-General Subjects 2017, 1861 (5), 1414-1428.

81. Soundararajan, S.; Chen, W.; Spicer, E. K.; Courtenay-Luck, N.; Fernandes, D. J., The nucleolin targeting aptamer AS1411 destabilizes Bcl-2 messenger RNA in human breast cancer cells. Cancer research 2008, 68 (7), 2358-2365.

82. Soundararajan, S.; Wang, L.; Sridharan, V.; Chen, W.; Courtenay-Luck, N.; Jones, D.; Spicer, E. K.; Fernandes, D. J., Plasma membrane nucleolin is a receptor for the anticancer aptamer AS1411 in MV4-11 leukemia cells. Molecular pharmacology 2009, 76 (5), 984-991.

83. He, F.; Wen, N.; Xiao, D.; Yan, J.; Xiong, H.; Cai, S.; Liu, Z.; Liu, Y., Aptamer-based targeted drug delivery systems: current potential and challenges. Current medicinal chemistry 2020, 27 (13), 2189-2219.

84. Oroojalian, F.; Rezayan, A. H.; Shier, W. T.; Abnous, K.; Ramezani, M., Megalintargeted enhanced transfection efficiency in cultured human $\mathrm{HK}-2$ renal tubular proximal cells 
using aminoglycoside-carboxyalkyl-polyethylenimine-containing nanoplexes. International journal of pharmaceutics 2017, 523 (1), 102-120.

85. Li, X.; Yu, Y.; Ji, Q.; Qiu, L., Targeted delivery of anticancer drugs by aptamer AS1411 mediated Pluronic F127/cyclodextrin-linked polymer composite micelles. Nanomedicine: Nanotechnology, Biology and Medicine 2015, 11 (1), 175-184.

86. Liao, Z.-X.; Chuang, E.-Y.; Lin, C.-C.; Ho, Y.-C.; Lin, K.-J.; Cheng, P.-Y.; Chen, K.-J.; Wei, H.-J.; Sung, H.-W., An AS1411 aptamer-conjugated liposomal system containing a bubblegenerating agent for tumor-specific chemotherapy that overcomes multidrug resistance. Journal of controlled release 2015, 208, 42-51.

87. Peng, L.-H.; Zhang, Y.-H.; Han, L.-J.; Zhang, C.-Z.; Wu, J.-H.; Wang, X.-R.; Gao, J.-Q.; Mao, Z.-W., Cell membrane capsules for encapsulation of chemotherapeutic and cancer cell targeting in vivo. ACS Applied Materials \& Interfaces 2015, 7 (33), 18628-18637.

88. Mohammadzadeh, P.; Cohan, R. A.; Ghoreishi, S. M.; Bitarafan-Rajabi, A.; Ardestani, M. S., AS1411 aptamer-anionic linear globular dendrimer G2-lohexol selective nanotheranostics. Scientific reports 2017, 7 (1), 1-16.

89. Torchilin, P. V.; Torchilin, V.; Torchilin, V.; Weissig, V., Liposomes: a practical approach. Oxford University Press: 2003.

90. Chen, W.; Goldys, E. M.; Deng, W., Light-induced liposomes for cancer therapeutics. Progress in Lipid Research 2020, 101052.

91. Lee, M.-K., Liposomes for Enhanced Bioavailability of Water-Insoluble Drugs: In Vivo Evidence and Recent Approaches. Pharmaceutics 2020, 12 (3), 264.

92. Bulbake, U.; Doppalapudi, S.; Kommineni, N.; Khan, W., Liposomal formulations in clinical use: an updated review. Pharmaceutics 2017, 9 (2), 12.

93. GrÜnweiler, A.; Hartmann, R. K., Locked nucleic acid oligonucleotides. BioDrugs 2007, 21 (4), 235-243.

94. Jiang, L.; Wang, H.; Chen, S., Aptamer (AS1411)-Conjugated Liposome for Enhanced Therapeutic Efficacy of miRNA-29b in Ovarian Cancer. Journal of nanoscience and nanotechnology 2020, 20 (4), 2025-2031.

95. Olusanya, T. O.; Haj Ahmad, R. R.; Ibegbu, D. M.; Smith, J. R.; Elkordy, A. A., Liposomal drug delivery systems and anticancer drugs. Molecules 2018, 23 (4), 907.

96. Humber, C.; Tierney, J.; Symonds, R.; Collingwood, M.; Kirwan, J.; Williams, C.; Green, J., Chemotherapy for advanced, recurrent or metastatic endometrial cancer: a systematic review of Cochrane collaboration. Annals of Oncology 2007, 18 (3), 409-420.

97. Dam, D. H. M.; Lee, J. H.; Sisco, P. N.; Co, D. T.; Zhang, M.; Wasielewski, M. R.; Odom, T. W., Direct observation of nanoparticle-cancer cell nucleus interactions. ACS nano 2012, 6 (4), 3318-3326.

98. Li, X.; Wu, X.; Yang, H.; Li, L.; Ye, Z.; Rao, Y., A nuclear targeted Dox-aptamer loaded liposome delivery platform for the circumvention of drug resistance in breast cancer. Biomedicine \& Pharmacotherapy 2019, 117, 109072.

99. Yu, S.; Bi, X.; Yang, L.; Wu, S.; Yu, Y.; Jiang, B.; Zhang, A.; Lan, K.; Duan, S., Co-delivery of paclitaxel and PLK1-targeted siRNA using aptamer-functionalized cationic liposome for synergistic anti-breast cancer effects in vivo. Journal of biomedical nanotechnology 2019, 15 (6), 1135-1148.

100. Devulapally, R.; Paulmurugan, R., Polymer nanoparticles for drug and small silencing RNA delivery to treat cancers of different phenotypes. Wiley Interdisciplinary Reviews: Nanomedicine and Nanobiotechnology 2014, 6 (1), 40-60. 
101. Sharma, S.; Parmar, A.; Kori, S.; Sandhir, R., PLGA-based nanoparticles: a new paradigm in biomedical applications. TrAC trends in analytical chemistry 2016, 80, 30-40.

102. Tabatabaei Mirakabad, F. S.; Nejati-Koshki, K.; Akbarzadeh, A.; Yamchi, M. R.; Milani, M.; Zarghami, N.; Zeighamian, V.; Rahimzadeh, A.; Alimohammadi, S.; Hanifehpour, Y., PLGAbased nanoparticles as cancer drug delivery systems. Asian Pacific Journal of Cancer Prevention 2014, 15 (2), 517-535.

103. Jokerst, J. V.; Lobovkina, T.; Zare, R. N.; Gambhir, S. S., Nanoparticle PEGylation for imaging and therapy. Nanomedicine 2011, 6 (4), 715-728.

104. Otsuka, H.; Nagasaki, Y.; Kataoka, K., PEGylated nanoparticles for biological and pharmaceutical applications. Advanced drug delivery reviews 2003, 55 (3), 403-419.

105. Suh, J.; Choy, K.-L.; Lai, S. K.; Suk, J. S.; Tang, B. C.; Prabhu, S.; Hanes, J., PEGylation of nanoparticles improves their cytoplasmic transport. International journal of nanomedicine 2007, 2 (4), 735.

106. Alibolandi, M.; Ramezani, M.; Abnous, K.; Hadizadeh, F., AS1411 aptamer-decorated biodegradable polyethylene glycol-poly (lactic-co-glycolic acid) nanopolymersomes for the targeted delivery of gemcitabine to non-small cell lung cancer in vitro. Journal of pharmaceutical sciences 2016, 105 (5), 1741-1750.

107. Hayashi, H.; Kurata, T.; Nakagawa, K., Gemcitabine: efficacy in the treatment of advanced stage nonsquamous non-small cell lung cancer. Clinical Medicine Insights: Oncology 2011, 5, CMO. S6252.

108. Vandghanooni, S.; Eskandani, M.; Barar, J.; Omidi, Y., AS1411 aptamer-decorated cisplatin-loaded poly (lactic-co-glycolic acid) nanoparticles for targeted therapy of miR-21inhibited ovarian cancer cells. Nanomedicine 2018, 13 (21), 2729-2758.

109. Althurwi, S. I.; Jun, Q. Y.; Beale, P.; Huq, F., Sequenced Combinations of Platinum Drugs and Selected Phytochemicals Towards Overcoming Drug Resistance in Ovarian Tumour Models. Preprints 2020.

110. Yu, X.; Chen, Y.; Tian, R.; Li, J.; Li, H.; Lv, T.; Yao, Q., miRNA-21 enhances chemoresistance to cisplatin in epithelial ovarian cancer by negatively regulating PTEN. Oncology letters 2017, 14 (2), 1807-1810.

111. Ananta, J. S.; Paulmurugan, R.; Massoud, T. F., Nanoparticle-delivered antisense microRNA-21 enhances the effects of temozolomide on glioblastoma cells. Molecular pharmaceutics 2015, 12 (12), 4509-4517.

112. Mosafer, J.; Abnous, K.; Tafaghodi, M.; Mokhtarzadeh, A.; Ramezani, M., In vitro and in vivo evaluation of anti-nucleolin-targeted magnetic PLGA nanoparticles loaded with doxorubicin as a theranostic agent for enhanced targeted cancer imaging and therapy. European journal of pharmaceutics and biopharmaceutics 2017, 113, 60-74.

113. Ashjari, M.; Khoee, S.; Mahdavian, A. R., A multiple emulsion method for loading 5fluorouracil into a magnetite-loaded nanocapsule: a physicochemical investigation. Polymer International 2012, 61 (5), 850-859.

114. Sun, X.-Y.; Liu, M.-C.; Chen, X.-L.; Lin, H.-C.; Liu, B.; Peng, Y.-B.; Zhang, L.-Y.; Shen, J.-L.; Zhao, P., A dual-targeted nucleic acid moiety decorated SPION nanoparticles for chemophotodynamic synergistic therapy. Journal of Luminescence 2019, 209, 387-397.

115. Chorny, M.; Fishbein, I.; Yellen, B. B.; Alferiev, I. S.; Bakay, M.; Ganta, S.; Adamo, R.; Amiji, M.; Friedman, G.; Levy, R. J., Targeting stents with local delivery of paclitaxel-loaded magnetic nanoparticles using uniform fields. Proceedings of the National Academy of Sciences 2010, 107 (18), 8346-8351. 
116. Chorny, M.; Polyak, B.; Fishbein, I.; Alferiev, I.; Levy, R. J., Magnetically-driven biodegradable gene delivery nanoparticles formulated with surface-attached polycationic complex. Google Patents: 2010.

117. Jia, H.-Z.; Wang, W.; Zheng, D.-W.; Wang, X.; Yu, W.-Y.; Li, S.-Y.; Zhuo, R.-X.; Zhao, Y.-F.; Feng, J.; Zhang, X.-Z., Multifunctional nanotherapeutics with all-in-one nanoentrapment of drug/gene/inorganic nanoparticle. ACS applied materials \& interfaces 2016, 8 (11), 67846789.

118. Misra, S. K.; Schwartz-Duval, A. S.; Pan, D., Genomic DNA interactions mechanize peptidotoxin-mediated anticancer nanotherapy. Molecular Pharmaceutics 2017, 14 (7), 22542261.

119. Lale, S. V.; RG, A.; Aravind, A.; Kumar, D. S.; Koul, V., AS1411 aptamer and folic acid functionalized pH-responsive ATRP fabricated PPEGMA-PCL-pPEGMA polymeric nanoparticles for targeted drug delivery in cancer therapy. Biomacromolecules 2014, 15 (5), 1737-1752.

120. Zhao, P.; Jin, S.-f.; Lu, J.-Z.; Lv, J.-I.; Wu, G.-q.; Chen, P.-P.; Tan, C.-L.; Chen, D.-W., Novel porphyrin-daunomycin hybrids: Synthesis and preferential binding to G-quadruplexes over i-motif. Spectrochimica Acta Part A: Molecular and Biomolecular Spectroscopy 2015, 137, 227-235.

121. Gissberg, O.; Zaghloul, E. M.; Lundin, K. E.; Nguyen, C.-H.; Landras-Guetta, C.; Wengel, J.; Zain, R.; Smith, C. E., Delivery, effect on cell viability, and plasticity of modified aptamer constructs. nucleic acid therapeutics 2016, 26 (3), 183-189.

122. Taghavi, S.; Nia, A. H.; Abnous, K.; Ramezani, M., Polyethylenimine-functionalized carbon nanotubes tagged with AS1411 aptamer for combination gene and drug delivery into human gastric cancer cells. International journal of pharmaceutics 2017, 516 (1-2), 301-312.

123. Jin-Song, Y.; Zhao-Xia, W.; Cheng-Yu, L.; Xiao-Di, L.; Ming, S.; Yuan-Yuan, G.; Wei, D., Prognostic significance of $\mathrm{Bcl}-\mathrm{xL}$ gene expression in human colorectal cancer. Acta histochemica 2011, 113 (8), 810-814.

124. Pantarotto, D.; Singh, R.; McCarthy, D.; Erhardt, M.; Briand, J. P.; Prato, M.; Kostarelos, K.; Bianco, A., Functionalized carbon nanotubes for plasmid DNA gene delivery. Angewandte Chemie 2004, 116 (39), 5354-5358.

125. Varkouhi, A. K.; Foillard, S.; Lammers, T.; Schiffelers, R. M.; Doris, E.; Hennink, W. E.; Storm, G., SiRNA delivery with functionalized carbon nanotubes. International journal of pharmaceutics 2011, 416 (2), 419-425.

126. Adeli, M.; Soleyman, R.; Beiranvand, Z.; Madani, F., Carbon nanotubes in cancer therapy: a more precise look at the role of carbon nanotube-polymer interactions. Chemical Society Reviews 2013, 42 (12), 5231-5256.

127. Saraiva, C.; Praça, C.; Ferreira, R.; Santos, T.; Ferreira, L.; Bernardino, L., Nanoparticlemediated brain drug delivery: overcoming blood-brain barrier to treat neurodegenerative diseases. Journal of Controlled Release 2016, 235, 34-47.

128. Gao, H., Perspectives on dual targeting delivery systems for brain tumors. Journal of Neuroimmune Pharmacology 2017, 12 (1), 6-16.

129. Miranda, A.; Blanco-Prieto, M. J.; Sousa, J.; Pais, A.; Vitorino, C., Breaching barriers in glioblastoma. Part II: Targeted drug delivery and lipid nanoparticles. International Journal of Pharmaceutics 2017, 531 (1), 389-410.

130. Ito, S.; Ohtsuki, S.; Terasaki, T., Functional characterization of the brain-to-blood efflux clearance of human amyloid- $\beta$ peptide (1-40) across the rat blood-brain barrier. Neuroscience research 2006, 56 (3), 246-252. 
131. Maletínská, L.; Blakely, A., E.; A Bjornstad, K.; Deen, DF; Knoff, L; Forte, TM Human glioblastoma cell lines: Levels of low-density lipoprotein receptor and low-density lipoprotein receptor-related protein. Cancer Res 2000, 60, 2300-2303.

132. Wang, S.; Zhao, C.; Liu, P.; Wang, Z.; Ding, J.; Zhou, W., Facile construction of dualtargeting delivery system by using lipid capped polymer nanoparticles for anti-glioma therapy. RSC advances 2018, 8 (1), 444-453.

133. Li, Y.; Li, C.; Jiang, T.; Zhou, L.; Zhang, P.; Kwok, R. T.; Lam, J. W. Y.; Gong, P.; Cai, L.; Tang, B. Z., Aptamer-Anchored Rubrene-Loaded Organic Nanoprobes for Cancer Cell Targeting and Imaging. CCS Chemistry 2019, 1 (3), 251-260.

134. Ntziachristos, V., Going deeper than microscopy: the optical imaging frontier in biology. Nature methods 2010, 7 (8), 603-614.

135. Tsoi, K. M.; Dai, Q.; Alman, B. A.; Chan, W. C., Are quantum dots toxic? Exploring the discrepancy between cell culture and animal studies. Accounts of chemical research 2013, 46 (3), 662-671.

136. Winnik, F. M.; Maysinger, D., Quantum dot cytotoxicity and ways to reduce it. Accounts of chemical research 2013, 46 (3), 672-680.

137. Porkka, K.; Laakkonen, P.; Hoffman, J. A.; Bernasconi, M.; Ruoslahti, E., A fragment of the HMGN2 protein homes to the nuclei of tumor cells and tumor endothelial cells in vivo. Proceedings of the National Academy of Sciences 2002, 99 (11), 7444-7449.

138. Christian, S.; Pilch, J.; Akerman, M. E.; Porkka, K.; Laakkonen, P.; Ruoslahti, E., Nucleolin expressed at the cell surface is a marker of endothelial cells in angiogenic blood vessels. The Journal of cell biology 2003, 163 (4), 871-878.

139. Feng, X.; Jiang, D.; Kang, T.; Yao, J.; Jing, Y.; Jiang, T.; Feng, J.; Zhu, Q.; Song, Q.; Dong, N., Tumor-homing and penetrating peptide-functionalized photosensitizer-conjugated PEG-PLA nanoparticles for chemo-photodynamic combination therapy of drug-resistant cancer. ACS applied materials \& interfaces 2016, 8 (28), 17817-17832.

140. Lam, P. Y.; Hillyar, C. R.; Able, S.; Vallis, K. A., Synthesis and evaluation of an 18Flabeled derivative of F3 for targeting surface-expressed nucleolin in cancer and tumor endothelial cells. Journal of Labelled Compounds and Radiopharmaceuticals 2016, 59 (12), 492-499.

141. McNeeley, K. M.; Karathanasis, E.; Annapragada, A. V.; Bellamkonda, R. V., Masking and triggered unmasking of targeting ligands on nanocarriers to improve drug delivery to brain tumors. Biomaterials 2009, 30 (23-24), 3986-3995.

142. Peer, D.; Karp, J. M.; Hong, S.; Farokhzad, O. C.; Margalit, R.; Langer, R., Nanocarriers as an emerging platform for cancer therapy. Nature nanotechnology 2007, 2 (12), 751-760.

143. Bhagat, M.; Halligan, S.; Sofou, S., Nanocarriers to solid tumors: considerations on tumor penetration and exposure of tumor cells to therapeutic agents. Current pharmaceutical biotechnology 2012, 13 (7), 1306-1316.

144. Minchinton, A. I.; Tannock, I. F., Drug penetration in solid tumours. Nature Reviews Cancer 2006, 6 (8), 583-592.

145. Ruoslahti, E., Peptides as targeting elements and tissue penetration devices for nanoparticles. Advanced materials 2012, 24 (28), 3747-3756.

146. Sugahara, K. N.; Teesalu, T.; Karmali, P. P.; Kotamraju, V. R.; Agemy, L.; Girard, O. M.; Hanahan, D.; Mattrey, R. F.; Ruoslahti, E., Tissue-penetrating delivery of compounds and nanoparticles into tumors. Cancer cell 2009, 16 (6), 510-520. 
147. Roth, L.; Agemy, L.; Kotamraju, V.; Braun, G.; Teesalu, T.; Sugahara, K.; Hamzah, J.; Ruoslahti, E., Transtumoral targeting enabled by a novel neuropilin-binding peptide. Oncogene 2012, 31 (33), 3754-3763.

148. Li, Y.; Hao, L.; Liu, F.; Yin, L.; Yan, S.; Zhao, H.; Ding, X.; Guo, Y.; Cao, Y.; Li, P., Cell penetrating peptide-modified nanoparticles for tumor targeted imaging and synergistic effect of sonodynamic/HIFU therapy. International journal of nanomedicine 2019, 14, 5875.

149. Suehiro, S.; Ohnishi, T.; Yamashita, D.; Kohno, S.; Inoue, A.; Nishikawa, M.; Ohue, S.; Tanaka, J.; Kunieda, T., Enhancement of antitumor activity by using 5-ALA-mediated sonodynamic therapy to induce apoptosis in malignant gliomas: significance of high-intensity focused ultrasound on 5-ALA-SDT in a mouse glioma model. Journal of neurosurgery 2018, 129 (6), 1416-1428.

150. Deepagan, V.; You, D. G.; Um, W.; Ko, H.; Kwon, S.; Choi, K. Y.; Yi, G.-R.; Lee, J. Y.; Lee, D. S.; Kim, K., Long-circulating Au-TiO2 nanocomposite as a sonosensitizer for ROSmediated eradication of cancer. Nano letters 2016, 16 (10), 6257-6264.

151. Qian, X.; Zheng, Y.; Chen, Y., Micro/nanoparticle-augmented sonodynamic therapy (SDT): Breaking the depth shallow of photoactivation. Advanced materials 2016, 28 (37), 80978129.

152. Khdair, A.; Gerard, B.; Handa, H.; Mao, G.; Shekhar, M. P.; Panyam, J., Surfactantpolymer nanoparticles enhance the effectiveness of anticancer photodynamic therapy. Molecular pharmaceutics 2008, 5 (5), 795-807.

153. Qin, M.; Hah, H. J.; Kim, G.; Nie, G.; Lee, Y.-E. K.; Kopelman, R., Methylene blue covalently loaded polyacrylamide nanoparticles for enhanced tumor-targeted photodynamic therapy. Photochemical \& Photobiological Sciences 2011, 10 (5), 832-841.

154. Yu, J.; Nguyen, H. N. Y.; Steenbergen, W.; Kim, K., Recent Development of Technology and Application of. 2018.

155. Xu, G.; Qin, M.; Mukundan, A.; Siddiqui, J.; Takada, M.; Vilar-Saavedra, P.; Tomlins, S. A.; Kopelman, R.; Wang, X. In Prostate cancer characterization by optical contrast enhanced photoacoustics, Photons Plus Ultrasound: Imaging and Sensing 2016, International Society for Optics and Photonics: 2016; p 97080I.

156. Johannsen, M.; Gneveckow, U.; Thiesen, B.; Taymoorian, K.; Cho, C. H.; Waldöfner, N.; Scholz, R.; Jordan, A.; Loening, S. A.; Wust, P., Thermotherapy of prostate cancer using magnetic nanoparticles: feasibility, imaging, and three-dimensional temperature distribution. European urology 2007, 52 (6), 1653-1662.

157. Zhang, R.; Xiong, C.; Huang, M.; Zhou, M.; Huang, Q.; Wen, X.; Liang, D.; Li, C., Peptide-conjugated polymeric micellar nanoparticles for Dual SPECT and optical imaging of EphB4 receptors in prostate cancer xenografts. Biomaterials 2011, 32 (25), 5872-5879.

158. Wang, X.; Pang, Y.; Ku, G.; Xie, X.; Stoica, G.; Wang, L. V., Noninvasive laser-induced photoacoustic tomography for structural and functional in vivo imaging of the brain. Nature biotechnology 2003, 21 (7), 803-806.

159. Yuan, J.; Xu, G.; Yu, Y.; Zhou, Y.; Carson, P. L.; Wang, X.; Liu, X., Real-time photoacoustic and ultrasound dual-modality imaging system facilitated with graphics processing unit and code parallel optimization. Journal of biomedical optics 2013, 18 (8), 086001.

160. Diamantopoulou, Z.; Gilles, M.-E.; Sader, M.; Cossutta, M.; Vallée, B.; Houppe, C.; Habert, D.; Brissault, B.; Leroy, E.; Maione, F., Multivalent cationic pseudopeptide polyplexes as a tool for cancer therapy. Oncotarget 2017, 8 (52), 90108. 
161. Caruso, F., Hollow capsule processing through colloidal templating and self-assembly. Chemistry-A European Journal 2000, 6 (3), 413-419.

162. Peyratout, C. S.; Dahne, L., Tailor-made polyelectrolyte microcapsules: from multilayers to smart containers. Angew Chem Int Ed Engl 2004, 43 (29), 3762-83.

163. Mora-Huertas, C. E.; Fessi, H.; Elaissari, A., Polymer-based nanocapsules for drug delivery. International Journal of Pharmaceutics 2010, 385 (1), 113-142.

164. Caruso, F.; Caruso, R. A.; Möhwald, H., Nanoengineering of inorganic and hybrid hollow spheres by colloidal templating. Science 1998, 282 (5391), 1111-1114.

165. De Koker, S.; Hoogenboom, R.; De Geest, B. G., Polymeric multilayer capsules for drug delivery. Chem Soc Rev 2012, 41 (7), 2867-84.

166. Tong, W.; Song, X.; Gao, C., Layer-by-layer assembly of microcapsules and their biomedical applications. Chem Soc Rev 2012, 41 (18), 6103-24.

167. Johnston, A. P. R.; Cortez, C.; Angelatos, A. S.; Caruso, F., Layer-by-layer engineered capsules and their applications. Current Opinion in Colloid \& Interface Science 2006, 11 (4), 203-209.

168. Decher, G., Fuzzy nanoassemblies: toward layered polymeric multicomposites. science 1997, 277 (5330), 1232-1237.

169. Decher, G.; Hong, J., Thin Solid Films, 210-211, 831;(c) Decher. G.(1997) Science 1992, $277,1232$.

170. Dubas, S. T.; Schlenoff, J. B., Factors controlling the growth of polyelectrolyte multilayers. Macromolecules 1999, 32 (24), 8153-8160.

171. Büscher, K.; Graf, K.; Ahrens, H.; Helm, C. A., Influence of adsorption conditions on the structure of polyelectrolyte multilayers. Langmuir 2002, 18 (9), 3585-3591.

172. Tan, H. L.; McMurdo, M. J.; Pan, G.; Van Patten, P. G., Temperature dependence of polyelectrolyte multilayer assembly. Langmuir 2003, 19 (22), 9311-9314.

173. Halthur, T. J.; Elofsson, U. M., Multilayers of charged polypeptides as studied by in situ ellipsometry and quartz crystal microbalance with dissipation. Langmuir 2004, 20 (5), 17391745.

174. Klitzing, R. V.; Wong, J. E.; Jaeger, W.; Steitz, R., Short range interactions in polyelectrolyte multilayers. Current opinion in colloid \& interface science 2004, 9 (1-2), 158162.

175. Borges, J. o.; Mano, J. F., Molecular interactions driving the layer-by-layer assembly of multilayers. Chemical reviews 2014, 114 (18), 8883-8942.

176. Gil, P. R.; del Mercato, L. L.; del_Pino, P.; Muñoz_Javier, A.; Parak, W. J., Nanoparticlemodified polyelectrolyte capsules. Nano Today 2008, 3 (3), 12-21.

177. Yang, X.; Johnson, S.; Shi, J.; Holesinger, T.; Swanson, B., Polyelectrolyte and molecular host ion self-assembly to multilayer thin films: An approach to thin film chemical sensors. Sensors and Actuators B: Chemical 1997, 45 (2), 87-92.

178. Belbekhouche, S.; Charaabi, S.; Picton, L.; Le Cerf, D.; Carbonnier, B., Glucosesensitive polyelectrolyte microcapsules based on (alginate/chitosan) pair. Carbohydrate Polymers 2018, 184, 144-153.

179. Belbekhouche, S.; Charaabi, S.; Carbonnier, B., Glucose-sensitive capsules based on hydrogen-bonded (polyvinylpyrrolidone / phenylboronic -modified alginate) system. Colloids and Surfaces B: Biointerfaces 2019, 177, 416-424.

180. Belbekhouche, S.; Bousserrhine, N.; Alphonse, V.; Carbonnier, B., From betacyclodextrin polyelectrolyte to layer-by-layer self-assembly microcapsules: From inhibition of bacterial growth to bactericidal effect. Food Hydrocolloids 2019, 95, 219-227. 
181. Bunton, C. A.; Nome, F.; Quina, F. H.; Romsted, L. S., lon binding and reactivity at charged aqueous interfaces. Accounts of Chemical Research 1991, 24 (12), 357-364.

182. Abbas, S.; Karangwa, E.; Bashari, M.; Hayat, K.; Hong, X.; Sharif, H. R.; Zhang, X., Fabrication of polymeric nanocapsules from curcumin-loaded nanoemulsion templates by self-assembly. Ultrasonics sonochemistry 2015, 23, 81-92.

183. Beaman, D. K.; Robertson, E. J.; Richmond, G. L., Unique Assembly of Charged Polymers at the Oil- Water Interface. Langmuir 2011, 27 (6), 2104-2106.

184. Li, Y.; Hu, M.; Du, Y.; McClements, D. J., Controlling lipid nanoemulsion digestion using nanolaminated biopolymer coatings. Journal of microencapsulation 2011, 28 (3), 166-175.

185. Preetz, C.; Rübe, A.; Reiche, I.; Hause, G.; Mäder, K., Preparation and characterization of biocompatible oil-loaded polyelectrolyte nanocapsules. Nanomedicine: Nanotechnology, Biology and Medicine 2008, 4 (2), 106-114.

186. Szczepanowicz, K.; Dronka-Góra, D.; Para, G.; Warszyński, P., Encapsulation of liquid cores by layer-by-layer adsorption of polyelectrolytes. Journal of microencapsulation 2010, 27 (3), 198-204.

187. Becker, A. L.; Johnston, A. P.; Caruso, F., Layer-by-layer-assembled capsules and films for therapeutic delivery. Small 2010, 6 (17), 1836-52.

188. Gittins, D. I.; Caruso, F., Multilayered polymer nanocapsules derived from gold nanoparticle templates. Advanced Materials 2000, 12 (24), 1947-1949.

189. Gittins, D. I.; Caruso, F., Tailoring the Polyelectrolyte Coating of Metal Nanoparticles. The Journal of Physical Chemistry B 2001, 105 (29), 6846-6852.

190. Belbekhouche, S.; Bousserrhine, N.; Alphonse, V.; Le Floch, F.; Charif Mechiche, Y.; Menidjel, I.; Carbonnier, B., Chitosan based self-assembled nanocapsules as antibacterial agent. Colloids and Surfaces B: Biointerfaces 2019, 181, 158-165.

191. Belbekhouche, S.; Mansour, O.; Carbonnier, B., Promising sub-100 nm tailor made hollow chitosan/poly (acrylic acid) nanocapsules for antibiotic therapy. Journal of colloid and interface science 2018, 522, 183-190.

192. Belbekhouche, S.; Oniszczuk, J.; Pawlak, A.; El Joukhar, I.; Goffin, A.; Varrault, G.; Sahali, D.; Carbonnier, B., Cationic poly(cyclodextrin)/alginate nanocapsules: From design to application as efficient delivery vehicle of 4-hydroxy tamoxifen to podocyte in vitro. Colloids and Surfaces B: Biointerfaces 2019, 179, 128-135.

193. Mansour, O.; Peker, T.; Hamadi, S.; Belbekhouche, S., Glucose-Responsive Capsules Based on (Phenylboronic-modified Poly(Lysine)/Alginate) System. European Polymer Journal 2019, 120, 109248.

194. Belbekhouche, S.; Cossutta, M.; Habert, D.; Hamadi, S.; Modjinou, T.; Cascone, I.; Courty, J., N6L-functionalized nanoparticles for targeted and inhibited pancreatic cancer cells. Colloids and Surfaces A: Physicochemical and Engineering Aspects 2020, 607, 125461.

195. Marinakos, S. M.; Novak, J. P.; Brousseau, L. C.; House, A. B.; Edeki, E. M.; Feldhaus, J. C.; Feldheim, D. L., Gold particles as templates for the synthesis of hollow polymer capsules. Control of capsule dimensions and guest encapsulation. Journal of the American Chemical Society 1999, 121 (37), 8518-8522.

196. Sukhorukov, G. B.; Donath, E.; Lichtenfeld, H.; Knippel, E.; Knippel, M.; Budde, A.; Möhwald, H., Layer-by-layer self assembly of polyelectrolytes on colloidal particles. Colloids and Surfaces A: physicochemical and engineering aspects 1998, 137 (1-3), 253-266.

197. Mulvaney, P., Surface plasmon spectroscopy of nanosized metal particles. Langmuir 1996, 12 (3), 788-800. 
198. Bhattacharjee, R. R.; Chakraborty, M.; Mandal, T. K., Synthesis of dendrimer-stabilized gold-polypyrrole core-shell nanoparticles. Journal of nanoscience and nanotechnology 2003, 3 (6), 487-491.

199. Poostforooshan, J.; Belbekhouche, S.; Shaban, M.; Alphonse, V.; Habert, D.; Bousserrhine, N.; Courty, J.; Weber, A. P., Aerosol-Assisted Synthesis of Tailor-Made Hollow Mesoporous Silica Microspheres for Controlled Release of Antibacterial and Anticancer Agents. ACS Applied Materials \& Interfaces 2020, 12 (6), 6885-6898.

200. Sader, M.; Couleaud, P.; Carpentier, G.; Gilles, M.-E.; Bousserrhine, N.; Livet, A.; Cascone, I.; Destouches, D.; Cortajarena, A. L.; Courty, J., Functionalization of iron oxide magnetic nanoparticles with the multivalent pseudopeptide $\mathrm{N} 6 \mathrm{I}$ for breast tumor targeting. Journal of Nanomedicine \& Nanotechnology 2015, 6 (4), 1.

201. Diamantopoulou, Z.; Gilles, M. E.; Sader, M.; Cossutta, M.; Vallee, B.; Houppe, C.; Habert, D.; Brissault, B.; Leroy, E.; Maione, F.; Giraudo, E.; Destouches, D.; Penelle, J.; Courty, J.; Cascone, I., Multivalent cationic pseudopeptide polyplexes as a tool for cancer therapy. Oncotarget 2017, 8 (52), 90108-90122.

202. Jose, J.; Kumar, R.; Harilal, S.; Mathew, G. E.; Parambi, D. G. T.; Prabhu, A.; Uddin, M. S.; Aleya, L.; Kim, H.; Mathew, B., Magnetic nanoparticles for hyperthermia in cancer treatment: an emerging tool. Environ Sci Pollut Res Int 2020, 27 (16), 19214-19225.

203. Kumar, C. S.; Mohammad, F., Magnetic nanomaterials for hyperthermia-based therapy and controlled drug delivery. Adv Drug Deliv Rev 2011, 63 (9), 789-808.

204. Sohail, A.; Ahmad, Z.; Beg, O. A.; Arshad, S.; Sherin, L., A review on hyperthermia via nanoparticle-mediated therapy. Bull Cancer 2017, 104 (5), 452-461.

205. Vangijzegem, T.; Stanicki, D.; Laurent, S., Magnetic iron oxide nanoparticles for drug delivery: applications and characteristics. Expert Opin Drug Deliv 2019, 16 (1), 69-78.

206. Wu, K.; Su, D.; Liu, J.; Saha, R.; Wang, J. P., Magnetic nanoparticles in nanomedicine: a review of recent advances. Nanotechnology 2019, 30 (50), 502003.

207. Avolio, M.; Gavilan, H.; Mazario, E.; Brero, F.; Arosio, P.; Lascialfari, A.; Puerto Morales, M., Elongated magnetic nanoparticles with high-aspect ratio: a nuclear relaxation and specific absorption rate investigation. Phys Chem Chem Phys 2019, 21 (34), 18741-18752. 208. Guo, X.; Wu, Z.; Li, W.; Wang, Z.; Li, Q.; Kong, F.; Zhang, H.; Zhu, X.; Du, Y. P.; Jin, Y., Appropriate size of magnetic nanoparticles for various bioapplications in cancer diagnostics and therapy. ACS Applied Materials \& Interfaces 2016, 8 (5), 3092-3106.

209. Nowak, J.; Wiekhorst, F.; Trahms, L.; Odenbach, S., The influence of hydrodynamic diameter and core composition on the magnetoviscous effect of biocompatible ferrofluids. Journal of Physics: Condensed Matter 2014, 26 (17), 176004.

210. Murph, S. E.; Larsen, G. K.; Lascola, R. J., Multifunctional Hybrid Fe2O3-Au Nanoparticles for Efficient Plasmonic Heating. J Vis Exp 2016, (108), 53598.

211. Peng, H.; Huang, Q.; Wu, T.; Wen, J.; He, H., Preparation of Porous gammaFe2O3@mWO3 Multifunctional Nanoparticles for Drug Loading and Controlled Release. Curr Drug Deliv 2018, 15 (2), 278-285.

212. Tang, S. C.; Lo, I. M., Magnetic nanoparticles: essential factors for sustainable environmental applications. Water Res 2013, 47 (8), 2613-32.

213. Veiseh, O.; Gunn, J. W.; Zhang, M., Design and fabrication of magnetic nanoparticles for targeted drug delivery and imaging. Adv Drug Deliv Rev 2010, 62 (3), 284-304.

214. Avval, Z. M.; Malekpour, L.; Raeisi, F.; Babapoor, A.; Mousavi, S. M.; Hashemi, S. A.; Salari, M., Introduction of magnetic and supermagnetic nanoparticles in new approach of targeting drug delivery and cancer therapy application. Drug Metab Rev 2020, 52 (1), 157-184. 
215. Hilger, I.; Kaiser, W. A., Iron oxide-based nanostructures for MRI and magnetic hyperthermia. Nanomedicine (Lond) 2012, 7 (9), 1443-59.

216. Ahmed, M.; Douek, M., The role of magnetic nanoparticles in the localization and treatment of breast cancer. Biomed Res Int 2013, 2013, 281230.

217. Srivastava, P.; Sharma, P. K.; Muheem, A.; Warsi, M. H., Magnetic Nanoparticles: A Review on Stratagems of Fabrication an d its Biomedical Applications. Recent Pat Drug Deliv Formul 2017, 11 (2), 101-113.

218. Kossatz, S.; Grandke, J.; Couleaud, P.; Latorre, A.; Aires, A.; Crosbie-Staunton, K.; Ludwig, R.; Dähring, H.; Ettelt, V.; Lazaro-Carrillo, A., Efficient treatment of breast cancer xenografts with multifunctionalized iron oxide nanoparticles combining magnetic hyperthermia and anti-cancer drug delivery. Breast Cancer Research 2015, 17 (1), 66.

219. Sanhaji, M.; Göring, J.; Couleaud, P.; Aires, A.; Cortajarena, A. L.; Courty, J.; PrinaMello, A.; Stapf, M.; Ludwig, R.; Volkov, Y., The phenotype of target pancreatic cancer cells influences cell death by magnetic hyperthermia with nanoparticles carrying gemicitabine and the pseudo-peptide NucAnt. Nanomedicine: Nanotechnology, Biology and Medicine 2019, 20, 101983. 


\section{Figure legend}

Figure 1 : Analysis of the number of publications and citations in the past ten years for the keywords : nucleolin, nanoparticles and cancer. (search engine Google Scholar).

Figure 2 : Elaboration of tailor-made particles via the templated self-assembly of particles. The advantages of this implemented strategy are that it is possible to control the size of the nanoparticles, the quantity of coated polymer, the thickness of the film, the mechanical properties and the loading and release of drugs.

Figure 3. Illustration of the multilayer films buildup driven by electrostatic interactions.

Figure 4. Schematic illustration of the polyelectrolytes adsorption process applied to the elaboration of N6L-based capsules.

Figure 5 :. $20 \mathrm{~nm}$ and $60 \mathrm{~nm}$ nanocapsules functionalized with N6L have been incubated with PANC-1 cells during $48 \mathrm{~h}$ and $72 \mathrm{~h}$. Cell viability has been quantified by alamar blue method as previously described ${ }^{48}$.

Figure 6 : Use of chitosan to design a pH-sensitive gatekeeper on the surface of hollow mesoporous silica microspheres

Figure 7. Controlled loading and release of N6L from the hollow mesoporous silica microspheres surface according to the $\mathrm{pH}$. 
Figure 1

Number of citations

(nucleolin, nanoparticles and cancer)

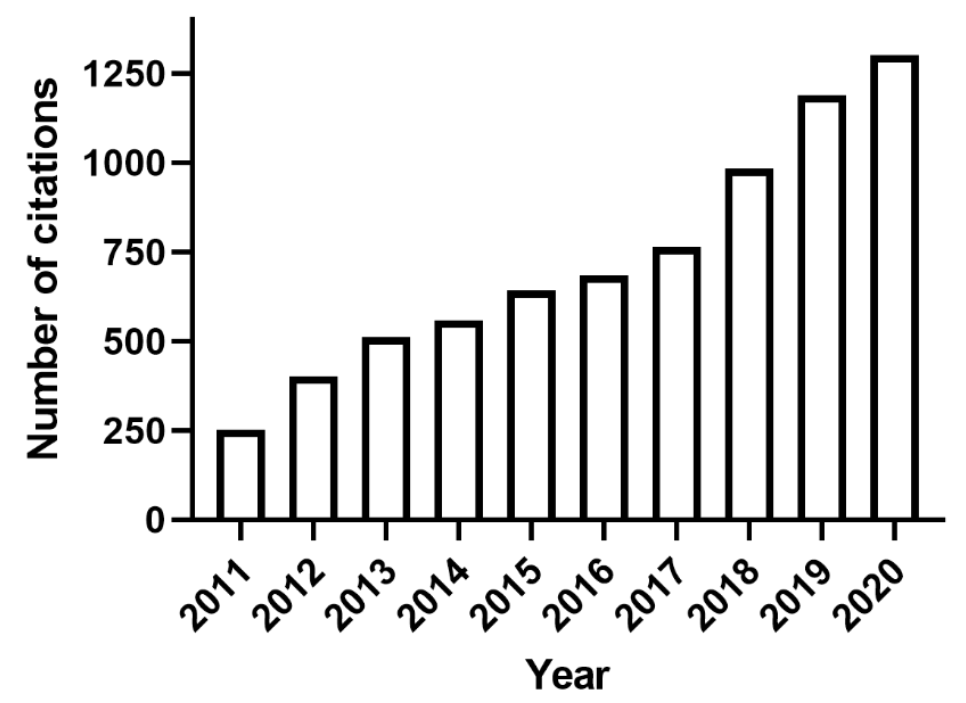


Figure 2

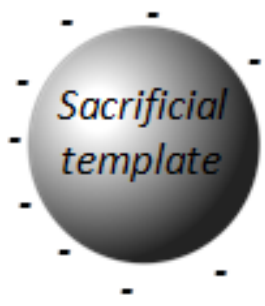

Liquid or solid

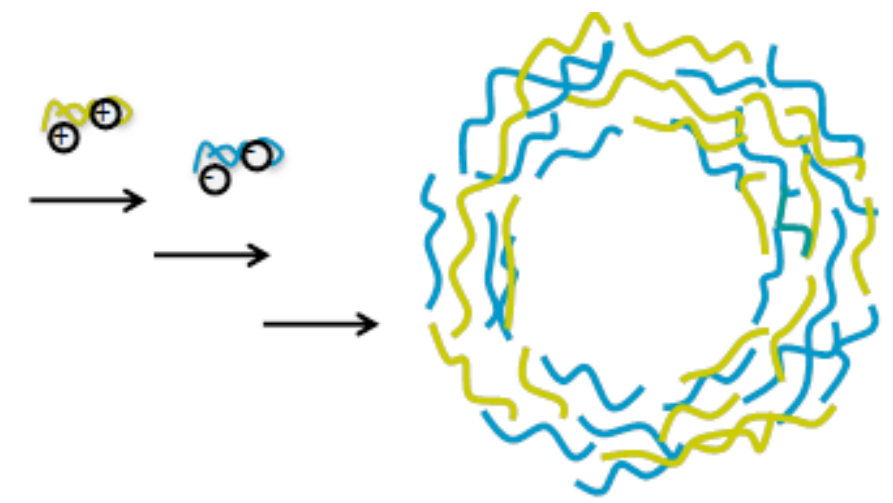


Figure 3

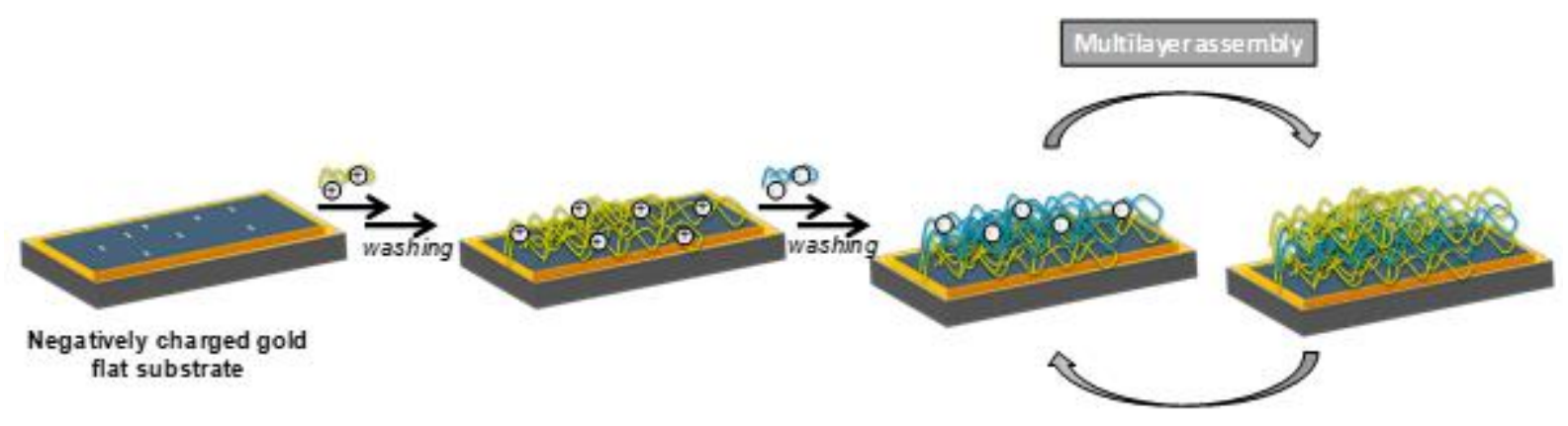


Figure 4

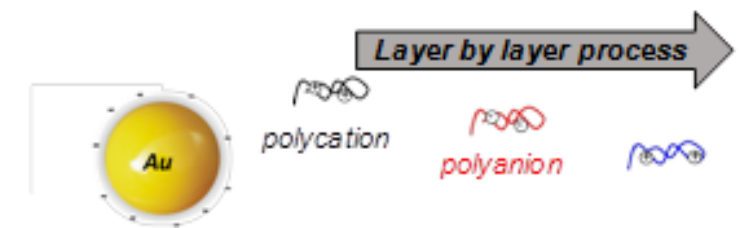

Goldnanoparticle

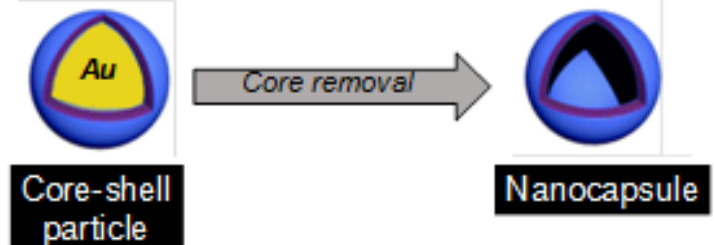
as te mplate 
Figure 5

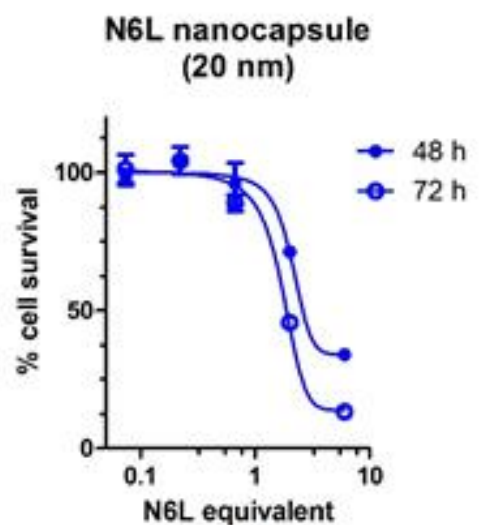

$(\mu \mathrm{M})$
N6L nanocapsule

(60 $\mathrm{nm}$ )

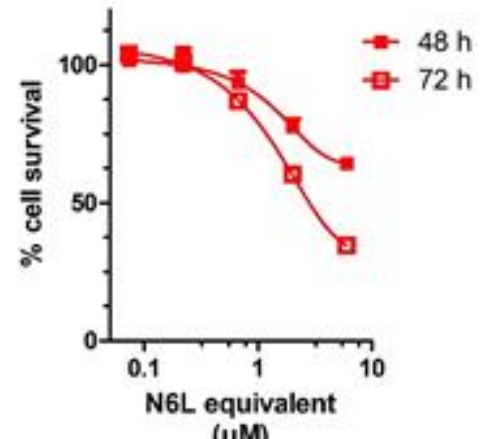


Figure 6

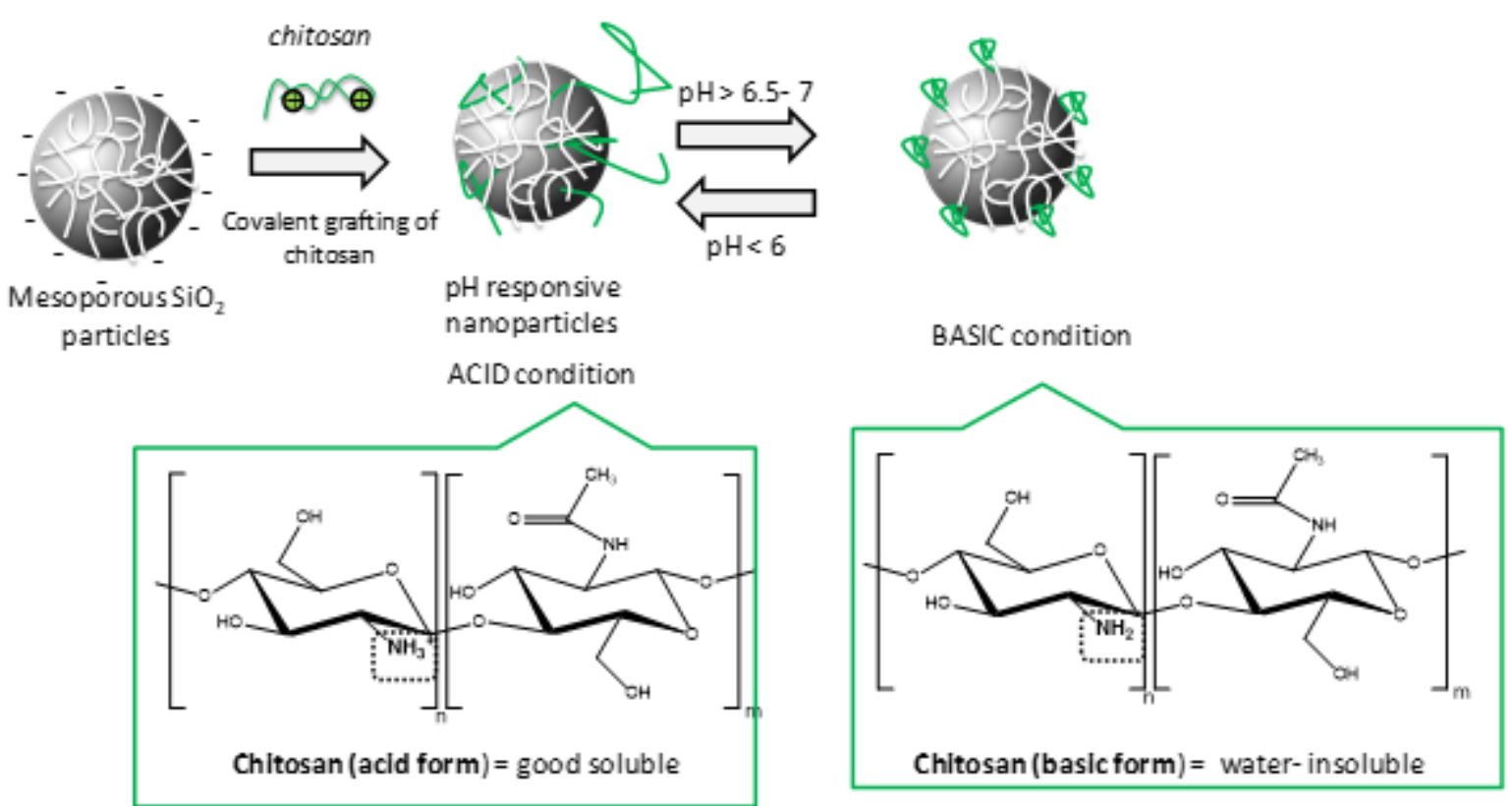


Figure 7

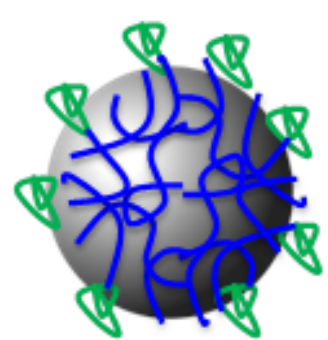

$\mathrm{pH}$ responsive nanoparticles

BASIC condition

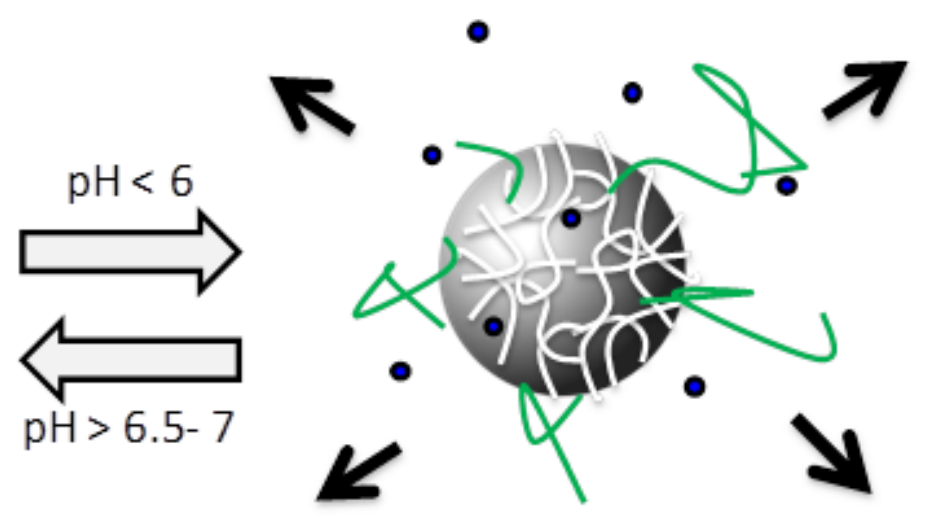

ACID condition

N6L Released 